\title{
PROTOTYPE OF 3D PRINTING ON RIBCAGE
}

\author{
GANDHASIRI RAHUL MOHAN, KOTA SAIDEEPAK, \\ PANDEM AVINASH REDDY, Mr. A. VIJAY KUMAR (Assistant Professor)
}

\author{
BACHELOR OF TECHNOLOGY \\ DEPARTMENT OF MECHANICAL ENGINEERING \\ GURUNANAK INSTITUTE OF TECHNOLOGY \\ (Affiliated to Jawaharlal Nehru Technological University) \\ Ibrahimpatnam, Ranga Reddy District-501506
}

\begin{abstract}
Medical 3D printing is emerging as a clinically relevant imaging tool in Directing preoperative and intraoperative planning in many surgical specialties and will therefore likely lead to interdisciplinary collaboration between engineers, radiologists, and surgeons. 3D printer technology is one of the innovations brought by the industrial age. It has been in our lives for many years. This miracle manufacturing method has been frequently preferred for medical applications in recent years. The main aim of this project is to minimise the time and cost for the design and manufacturing of the prosthesis. Getting the DICOM file from the MRI or CT scan which gives the body part dimensions (or) views of the body part in different directions. AUTODESK MESHMIXER and 3D SLICER software's are used to extract and design the 3D prosthetic body part in this project, flexible Biomaterial is gone to be used for the flexibility in the respiration process. There are a lot uses of 3D printing technology in surgery, pharmaceutical industry, disease modelling, development of customized implants and prostheses, organ printing, vet medicine and tissue engineering applications have been explained and this new method compared with traditional methods that used in the biomedical field. In addition, this study includes future opportunities that are expected to become widespread and developed in the future.
\end{abstract}

Keywords: 3D Printing, DICOM File, AUTODESK MESHMIXER, 3D Slicer. 


\section{INTRODUCTION}

\subsection{INTRODUCTION}

Nowadays, most schools are incorporating 3D printing methods into their curriculums. The assistance of 3D printing helps better prepare students for their future by allowing students to create prototypes without the need for expensive tooling. Students learn about 3D printing applications by designing and producing models they can actually hold.3D printing overcomes any issues among thoughts and pictures on a page or screen, considering the making of those thoughts/pictures in the physical, 3-dimensional world.3D printers are presently normally found in homerooms and public libraries. Colleges have 3D printers accessible for understudies to use in classes and activities. Organizations, most of the online learning platforms offers 3D printing courses and applications for the both lecturers and students.

3D printing tools are also revolutionizing STEM education by offering the ability for low-cost rapid prototyping by students in the classroom as well as fabricating low-cost high-quality scientific equipment from open hardware designs[1]

Students learn about a variety of 3D printing applications by exploring design, engineering, and architectural principles. They can duplicate museum items like fossils and historical artifacts to study in the classroom without the possibility of damaging delicate collections. They can gain a new, 3-dimensional perspective on topographic maps. Graphic design students can easily construct models with complex working parts. Students in the sciences can create and study cross-sections of organs in the human body as well as other biological specimens. Chemistry students can make 3D models of molecules and chemical compounds.

\subsection{HISTORY}

3D printing technology is not a new concept as many thinks. When FDM (fused deposition modelling) licenses expired in 2009, 3D printing became a new innovation topic. What's more, because of which it turned out to be more mainstream, individuals envisioned that FDM was just a single added substance producing system. Be that as it may, the initial 3D printing procedure was SLA not FDM, and its first patent was recorded in the 1980's. Here is the historical backdrop of 3D printing innovation, from 1980 to today the 3 primary 3D printing systems were introduced in 1980. Dr. Kodana was the first person to present a layer by-layer approach for assembling and furthermore he was the principal individual to create a fastprototyping strategy. He made a progenitor for SLA. He polymerized a photosensitive gum with the help of UV light, however, didn't succeed. Shockingly for Dr. Kodana, the complete patent detail wasn't recorded by him before the one-year maturity after the applianc. The causes of $3 \mathrm{~d}$ printing innovation are often followed from 1983. In 1983 Charles Hull was the person to patent stereolithography. Frame designed the term stereolithography in august 8, 1984 application for "Contraption for creation of three-dimensional questions by 3D Printing of stereolithography[2]. Furthermore, was the main individual to make the SLA1 (stereolithography) machine in 1987. 


\subsection{Types of 3D Printing}

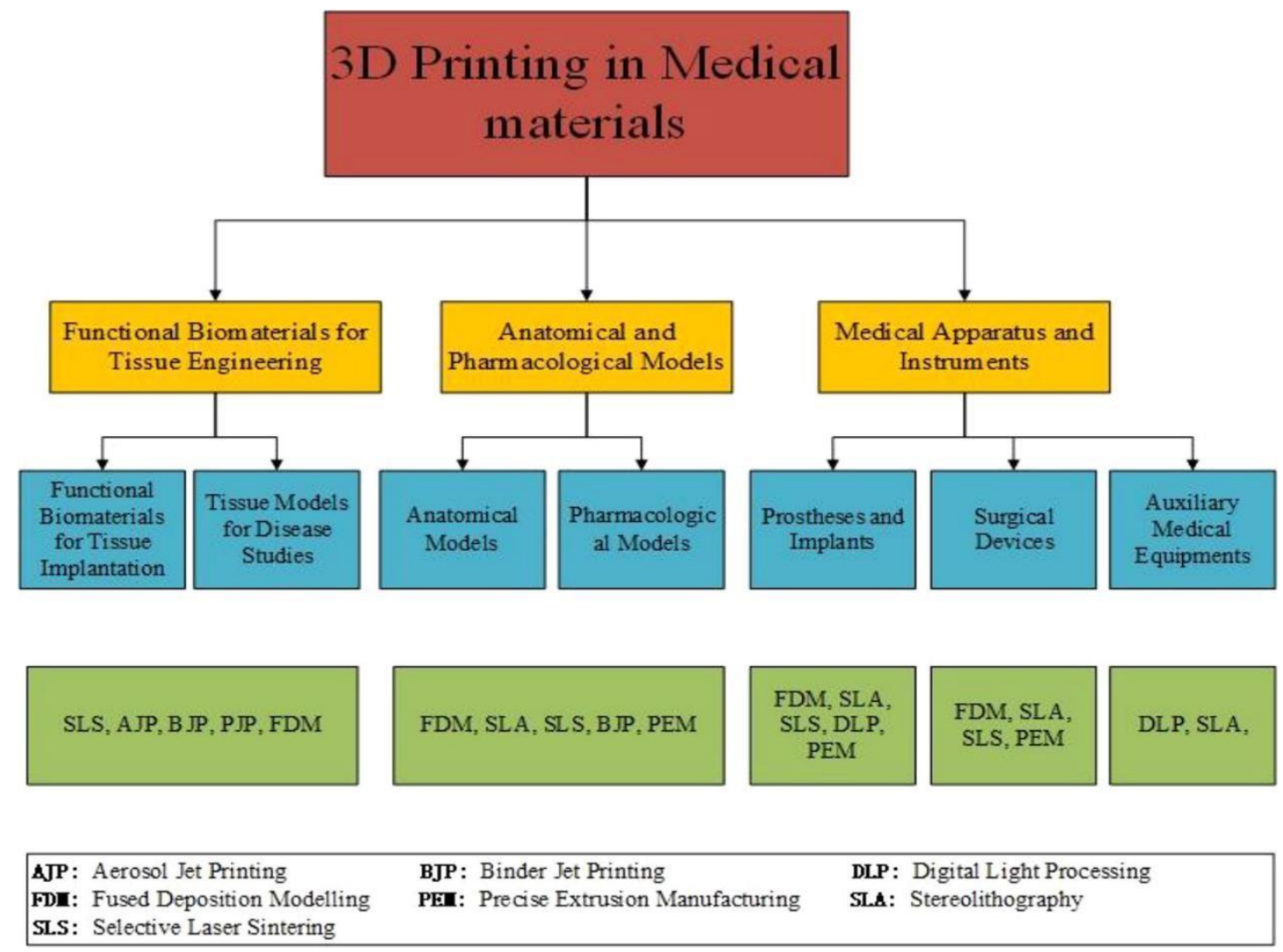

Fig.1. Types of 3D Printing

\subsubsection{Stereolithography (SLA):}

Stereolithography was the world's first 3D printing technology, invented within the 1980s, and remains one among the foremost popular technologies for professionals. SLA 3D printers use a laser to cure liquid resin into hardened plastic during a process called photopolymerization. SLA resin 3D printers became vastly popular for his or her ability to supply high-accuracy, isotropic, and watertight prototypes and parts during a range of advanced materials with fine features and smooth surface finish. SLA resin formulations offer a good range of optical, mechanical, and thermal properties to match those of ordinary, engineering, and industrial thermoplastics[3]

Resin 3D printing is an excellent option for highly detailed prototypes requiring tight tolerances and smooth surfaces, like moulds, patterns, and functional parts SLA 3D printers are widely utilized in a variety of industries from engineering and merchandise design to manufacturing, dentistry, jewellery, model making, and education. 


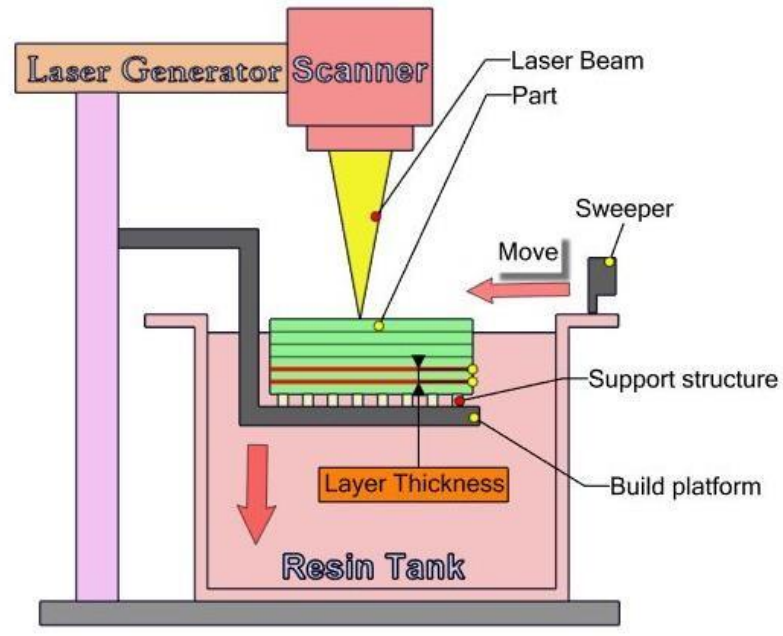

Fig.2. Stereolithography (SLA)

\subsubsection{Selective Laser Sintering (SLS):}

Selective laser sintering (SLS) 3D printers use a high-power laser to sinter small particles of polymer powder into a solid structure. The unfused powder supports the part during printing and eliminates the necessity for dedicated support structures. This makes SLS ideal for complex geometries, including interior features, undercuts, thin walls, and negative features. Parts produced with SLS printing have excellent mechanical characteristics, with strength resembling that of injection-molded parts. The most common material for selective laser sintering is nylon, a well-liked engineering thermoplastic with excellent mechanical properties. Nylon is lightweight, strong, and versatile, also as stable against impact, chemicals, heat, UV light, water, and dirt. The combination of low cost per part, high productivity, and established materials makes SLS a well-liked choice among engineers for functional prototyping, and an economical alternative to injection moulding for limited-run or bridge manufacturing[4]

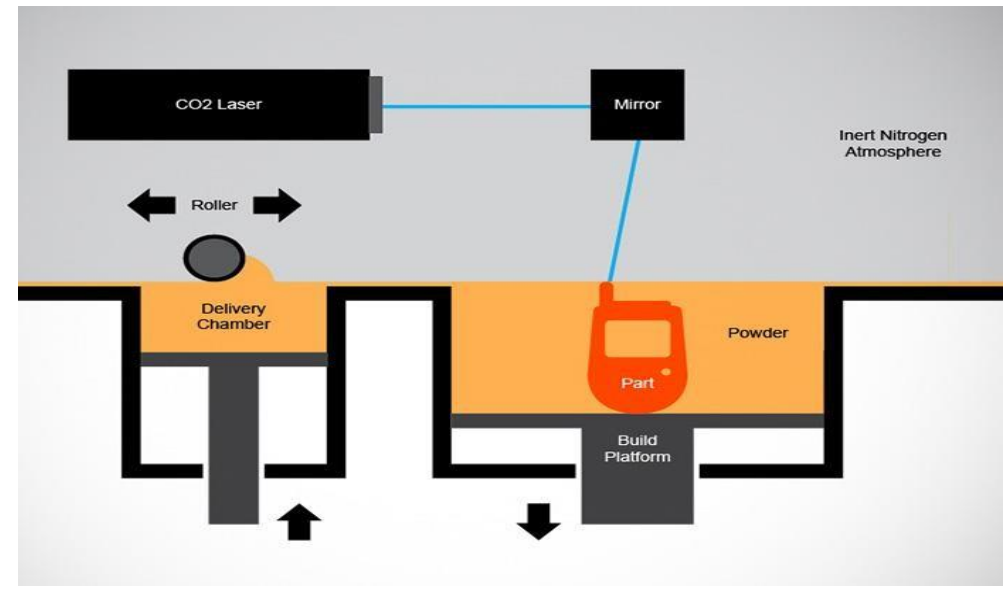

Fig.3. Selective Laser Sintering 


\subsubsection{Fused Deposition Modelling (FDM):}

Fused deposition modelling (FDM), also referred to as fused filament fabrication (FFF), is the most generally used sort of 3D printing at the buyer level. FDM 3D printers work by extruding thermoplastic filaments, like ABS (Acrylonitrile Butadiene Styrene), PLA (Polylactic Acid), through a heated nozzle, melting the fabric and applying the plastic layer by layer to a build platform. Each and every layer is laid down one at a time until the part is fully complete. FDM 3D printers are well-suited for basic proof-ofconcept models, also as quick and low-cost prototyping of straightforward parts, like parts which may typically be machined. However, FDM has rock bottom resolution and accuracy in comparison to SLA or SLS and isn't the simplest option for printing complex designs or parts with intricate features. High quality finishes could be obtained through chemical and mechanical polishing processes. Industrial FDM 3D printers use soluble supports to mitigate a number of these issues and offer a wider range of engineering thermoplastics, but they also come at a steep price[5]

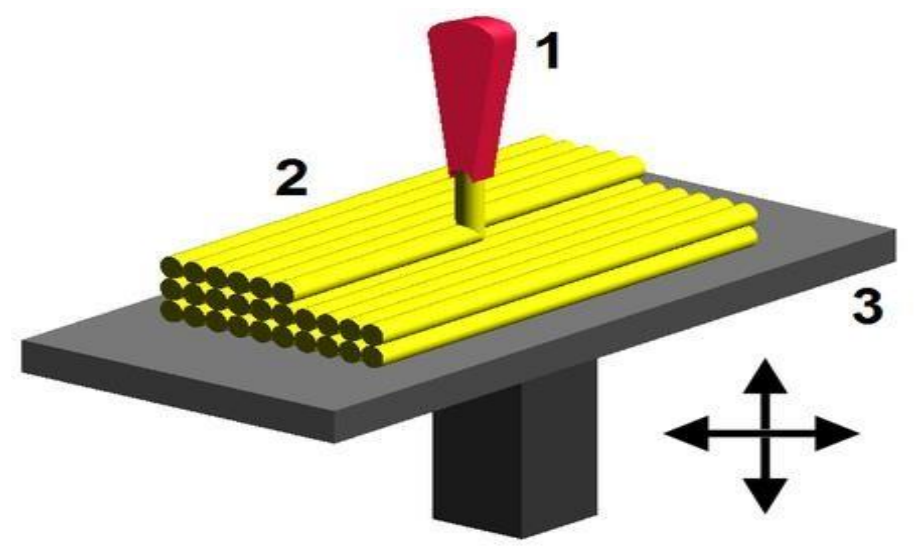

Fig.4 Layers of object material

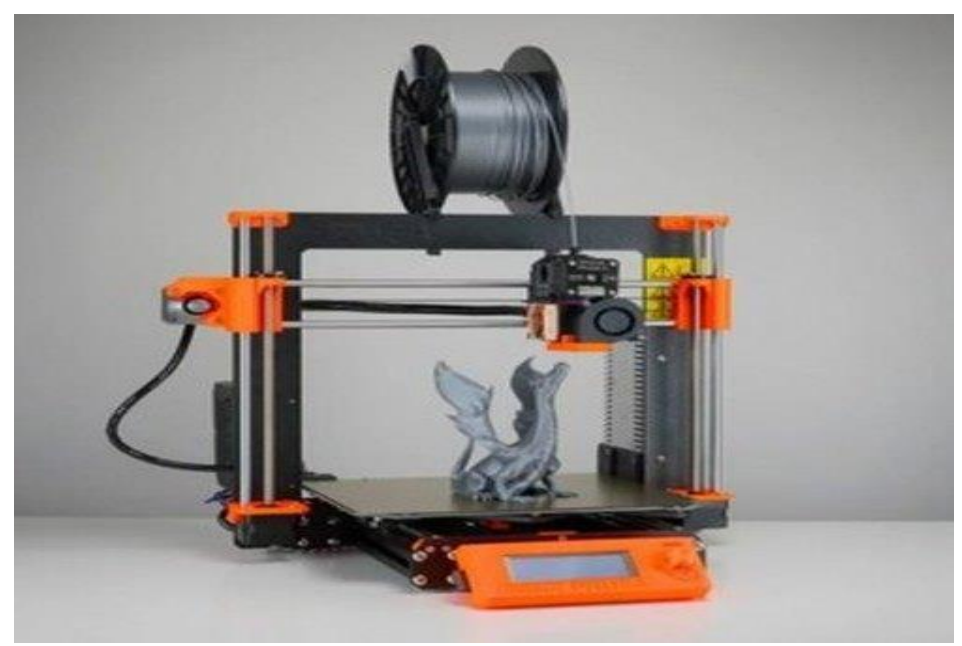

Fig.5 . Fused Deposition Modelling (FDM): 


\subsubsection{Multi Jet Fusion (MJF):}

Multi Jet Fusion assembles utilitarian parts from nylon powder. As against utilizing a laser to sinter the powder, MJF utilizes an inkjet cluster to use melding specialists to the bed of nylon powder. At that time, a warming component disregards the bed to mix each layer. This outcome in additional predictable mechanical properties contrasted with SLS even as improved surface completion. Another advantage of the MJF cycle is that the quickened fabricate time, which prompts lower creation costs. MJ differs from other sorts of 3D printing technologies that deposit, sinter, or cure build material with point-wise deposition. Instead, the print head jets many droplets of [6]photopolymer and cures/solidifies them using UV light. Once a layer is deposited and cured, the build platform lowers by one layer thickness, and therefore the process is repeated until the 3D object is made. Another difference from 3D printing technologies is that rather than employing a single point to follow a path that outlines the cross-sectional layer, MJ machines deposit build material during a fast, line-wise manner. Articles made with MJ need help during printing and are printed all the while during the shape cycle with a dissolvable material that's taken call at posthandling[7] $\mathrm{MJ}$ is one among the solitary kinds of 3D printing innovation which will make objects produced using numerous materials and with full tone. The advantage to the present is MJ printers can fabricate multiple objects during a single line without affecting build speed. As long as the models are arranged correctly with optimal spacing, MJ can produce parts faster than other sorts of 3D printers. Hence, there are multiple processes for multiple projects, selecting the simplest suitable process is of utmost importance.

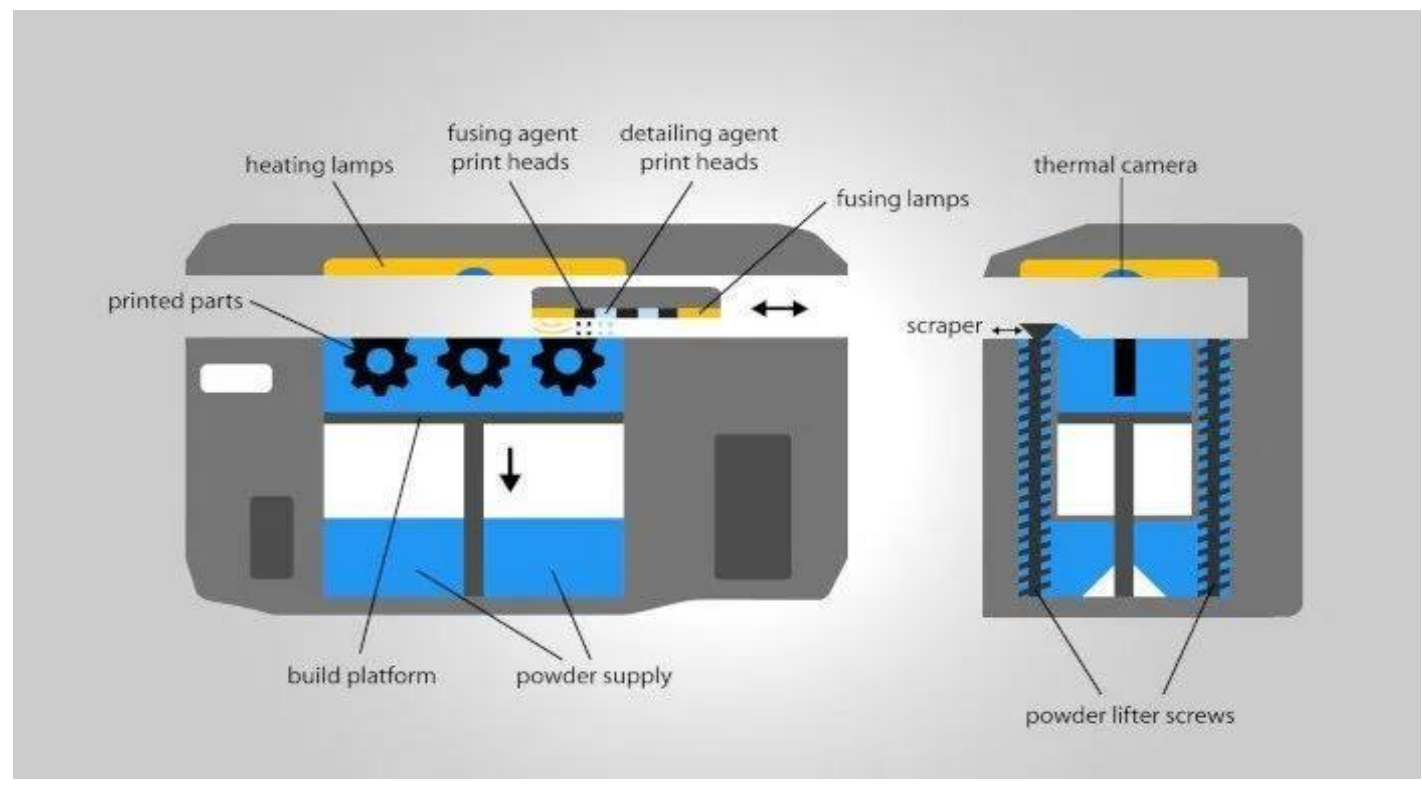

Fig.6 Multi Jet Fusion (MJF) 


\subsubsection{Direct Metal Laser Sintering (DMLS):}

Direct Metal Laser Sintering (DMLS) is an additive manufacturing technology that's based melting layers of metal powder using a powerful CO2 laser. The fact that layer-based production processes don't require tooling has led to the assumption that these technologies are free of rules that govern design for manufacture, like draft angles and undercuts.

Whilst DMLS doesn't need to observe conventional design rules, there are others that do apply to the method, particularly if reducing cost and waste may be a consideration[8]This presentation looks at these rules and how they affect the planning of parts built using the DMLS process.

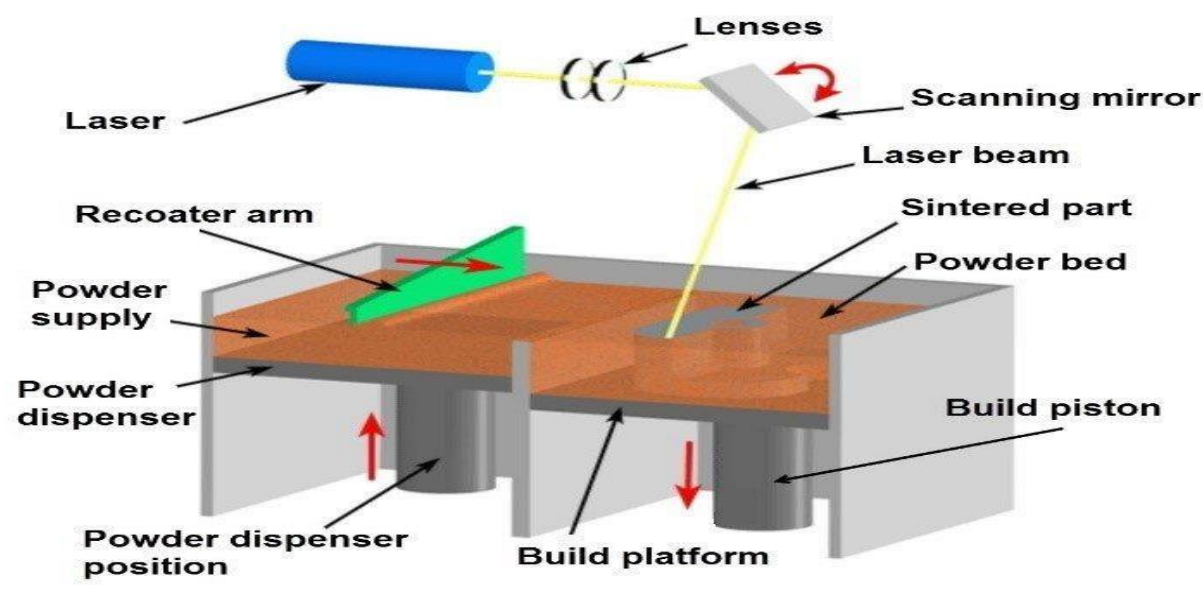

Fig.7. Direct Metal Laser Sintering (DMLS)

\subsection{Material and Types in 3D Printing:}

\begin{tabular}{|l|l|l|}
\hline \multicolumn{1}{|c|}{ Type } & \multicolumn{1}{|c|}{ Technologies } & \multicolumn{1}{c|}{ Materials } \\
\hline $\begin{array}{l}\text { Inkjet } \\
\text { Technology }\end{array}$ & $\begin{array}{l}\text { Drop-On-Demand or } \\
\text { Continuous (Single or Multi } \\
\text { nozzle) Particle Deposition }\end{array}$ & $\begin{array}{l}\text { Hot-melt materials (wax, } \\
\text { thermoplastic, metal alloy) }\end{array}$ \\
\hline $\begin{array}{l}\text { Material } \\
\text { extrusion }\end{array}$ & $\begin{array}{l}\text { Fused deposition } \\
\text { modelling (FDM) or Fused } \\
\text { filament fabrication (FFF) and } \\
\text { fused pellet fabrication or fused } \\
\text { particle fabrication }\end{array}$ & $\begin{array}{l}\text { Thermoplastics, eutectic metals, } \\
\text { edible materials, Rubbers, Modeling } \\
\text { clay, Plasticine }\end{array}$ \\
\hline
\end{tabular}




\begin{tabular}{|c|c|c|}
\hline & $\begin{array}{l}\text { Robocasting or MIG Welding } \\
\text { 3D Printing or Direct Ink } \\
\text { Writing (DIW) or Extrusion } \\
\text { based Additive Manufacturing } \\
\text { of Metals (EAM) and Ceramics } \\
\text { (EAC) }\end{array}$ & $\begin{array}{l}\text { Metal-binder mixtures } \\
\text { (including Metal clay and Precious } \\
\text { Metal Clay), ceramic-binder } \\
\text { mixtures (including ceramic clay } \\
\text { and ceramic slurries), cermet, metal } \\
\text { matrix composite, ceramic matrix } \\
\text { composite, Metal (MIG Welding) }\end{array}$ \\
\hline & $\begin{array}{l}\text { Composite Filament Fabrication } \\
(\mathrm{CFF})\end{array}$ & $\begin{array}{l}\text { Nylon or Nylon with short carbon } \\
\text { fiber + reinforcement in the form } \\
\text { Carbon, Kevlar, Glass and Glass for } \\
\text { high temperature fiber }\end{array}$ \\
\hline \multirow{3}{*}{$\begin{array}{l}\text { Light } \\
\text { polymerized }\end{array}$} & Stereolithography (SLA) & $\begin{array}{l}\text { Photopolymer (including preceramic } \\
\text { polymers) }\end{array}$ \\
\hline & Digital Light Processing (DLP) & Photopolymer \\
\hline & $\begin{array}{l}\text { Continuous Liquid Interface } \\
\text { Production (CLIP) }\end{array}$ & $\begin{array}{l}\text { Photopolymer }+ \text { thermally activated } \\
\text { chemistry }\end{array}$ \\
\hline \multirow{4}{*}{ Powder Bed } & $\begin{array}{l}\text { Powder bed and inkjet head 3D } \\
\text { printing (3DP) }\end{array}$ & $\begin{array}{l}\text { Almost any metal alloy, powdered } \\
\text { polymers, Plaster }\end{array}$ \\
\hline & Electron-beam melting (EBM) & $\begin{array}{l}\text { Almost any metal } \\
\text { alloy including Titanium alloys }\end{array}$ \\
\hline & Selective laser melting (SLM) & $\begin{array}{l}\text { Titanium alloys, Cobalt Chrome } \\
\text { alloys, Stainless Steel, Aluminium }\end{array}$ \\
\hline & Selective heat sintering (SHS) & Thermoplastic powder \\
\hline
\end{tabular}




\begin{tabular}{|c|c|c|}
\hline & Selective laser sintering (SLS) & $\begin{array}{l}\text { Thermoplastics, metal } \\
\text { powders, ceramic powders }\end{array}$ \\
\hline & $\begin{array}{l}\text { Direct metal laser } \\
\text { sintering (DMLS) }\end{array}$ & Almost any metal alloy \\
\hline Laminated & $\begin{array}{l}\text { Laminated object } \\
\text { manufacturing (LOM) }\end{array}$ & Paper, metal foil, plastic film \\
\hline Powder fed & Directed Energy Deposition & Almost any metal alloy \\
\hline Wire & $\begin{array}{l}\text { Electron beam freeform } \\
\text { fabrication }(\mathrm{EBF})\end{array}$ & Almost any metal alloy \\
\hline
\end{tabular}

Table.1. Material and Types in 3D Printing

\subsection{Advantages and Disadvantages on 3D printing Technology in the biomedical sector.}

\subsubsection{Pros of 3D Printing}

This production process offers a range of advantages compared to traditional manufacturing methods. These advantages include those related to design, time and cost, amongst others[9]

\section{i.Flexible Design}

3D printing allows for the design and print of more complex designs than traditional manufacturing processes. More traditional processes have design restrictions which no longer apply with the use of 3D printing[10]

\section{ii.Rapid Prototyping}

3D printing can manufacture parts within hours, which speeds up the prototyping process.

This allows for each stage to complete faster. When compared to machining prototypes, 3D printing is inexpensive and quicker at creating parts as the part can be finished in hours, allowing for each design modification to be completed at a much more efficient rate[11] 


\section{iii.Print on Demand}

Print on demand is another advantage as it doesn't need a lot of space to stock inventory, unlike traditional manufacturing processes. This saves space and costs as there is no need to print in bulk unless required.

The 3D design files are all stored in a virtual library as they are printed using a 3D model as either a CAD or STL file, this means they can be located and printed when needed. Edits to designs can be made at very low costs by editing individual files without wastage of out of-date inventory and investing in tools[12]

\section{iv.Strong and Lightweight Parts}

The main 3D printing material used is plastic, although some metals can also be used for 3D printing. However, plastics offer advantages as they are lighter than their metal equivalents. This is particularly important in industries such as automotive and aerospace where light-weighting is an issue and can deliver greater fuel efficiency. Also, parts can be created from tailored materials to provide specific properties such as heat resistance, higher strength or water repellence.

\section{v.Fast Design and Production}

Depending on a part's design and complexity, 3D printing can print objects within hours, which is much faster than moulded or machined parts. It is not only the manufacture of the part that can offer time savings through 3D printing but also the design process can be very quick by creating STL or CAD files ready to be printed.

\section{vi.Minimising Waste}

The production of parts only requires the materials needed for the part itself, with little or no wastage as compared to alternative methods which are cut from large chunks of nonrecyclable materials. Not only does the process save on resources but it also reduces the cost of the materials being used[13]

\section{vii.Cost Effective}

As a single step manufacturing process, 3D printing saves time and therefore costs associated with using different machines for manufacture. 3D printers can also be set up and left to get on with the job, meaning that there is no need for operators to be present the entire time. As mentioned above, this manufacturing process can also reduce costs on materials as it only uses the amount of material required for the part itself, with little or no wastage[14] While 3D printing equipment can be expensive to buy, you can even avoid this cost by outsourcing your project to a 3D printing service company. .

\section{viii.Environmentally Friendly}

As this technology reduces the amount of material wastage used this process is inherently environmentally friendly. However, the environmental benefits are extended when you consider factors such as improved fuel efficiency from using lightweight 3D printed parts[15]

\section{ix.Advanced Healthcare}

3D printing is being used in the medical sector to help save lives by printing organs for the human body such as livers, kidneys and hearts. Further advances and uses are being developed in the healthcare sector providing some of the biggest advances from using the technology. 


\subsubsection{Cons of 3D Printing}

Like with almost any other process there are also drawbacks of 3D printing technology which should be considered before opting to use this process.

\section{i. Limited Materials}

While 3D Printing can create items in a selection of plastics and metals the available selection of raw materials is not exhaustive. This is due to the fact that not all metals or plastics can be temperature controlled enough to allow 3D printing. In addition, many of these printable materials cannot be recycled and very few are food safe[16]

\section{ii. Restricted Build Size}

3D printers currently have small print chambers which restrict the size of parts that can be printed. Anything bigger will need to be printed in separate parts and joined together after production. This can increase costs and time for larger parts due to the printer needing to print more parts before manual labour is used to join the parts together.

\section{iii. Post Processing}

Although large parts require post-processing, as mentioned above, most 3D printed parts need some form of cleaning up to remove support material from the build and to smooth the surface to achieve the required finish. Post processing methods used include water jetting, sanding, a chemical soak and rinse, air or heat drying, assembly and others. The amount of post processing required depends on factors including the size of the part being produced, the intended application and the type of 3D printing technology used for production. So, while 3D printing allows for the fast production of parts, the speed of manufacture can be slowed by post processing.

\section{iv. Large Volumes}

3D printing is a static cost unlike more conventional techniques like injection moulding, where large volumes may be more cost effective to produce. While the initial investment for 3D printing may be lower than other manufacturing methods, once scaled up to produce large volumes for mass production, the cost per unit does not reduce as it would with injection moulding[17]

\section{V.Reduction in Manufacturing Jobs}

Another of the disadvantages of 3D technology is the potential reduction in human labour, since most of the production is automated and done by printers. However, many third world countries rely on low skill jobs to keep their economies running, and this technology could put these manufacturing jobs at risk by cutting out the need for production abroad.

\section{vi.Design Inaccuracies}

Another potential problem with 3D printing is directly related to the type of machine or process used, with some printers having lower tolerances, meaning that final parts may differ from the original design. This 
can be fixed in post processing, but it must be considered that this will further increase the time and cost of production

\subsection{PROCESS OF 3D PRINTING}

3D Printing encompasses various different technologies just like the Fused Deposition Modelling, Stereolithography, Digital Light Processing, Selective Laser Sintering, etc. but the essential principle of producing parts through a layer-by-layer process remains an equivalent for explanation purposes we showcase the working of an SLA 3D printing [18]

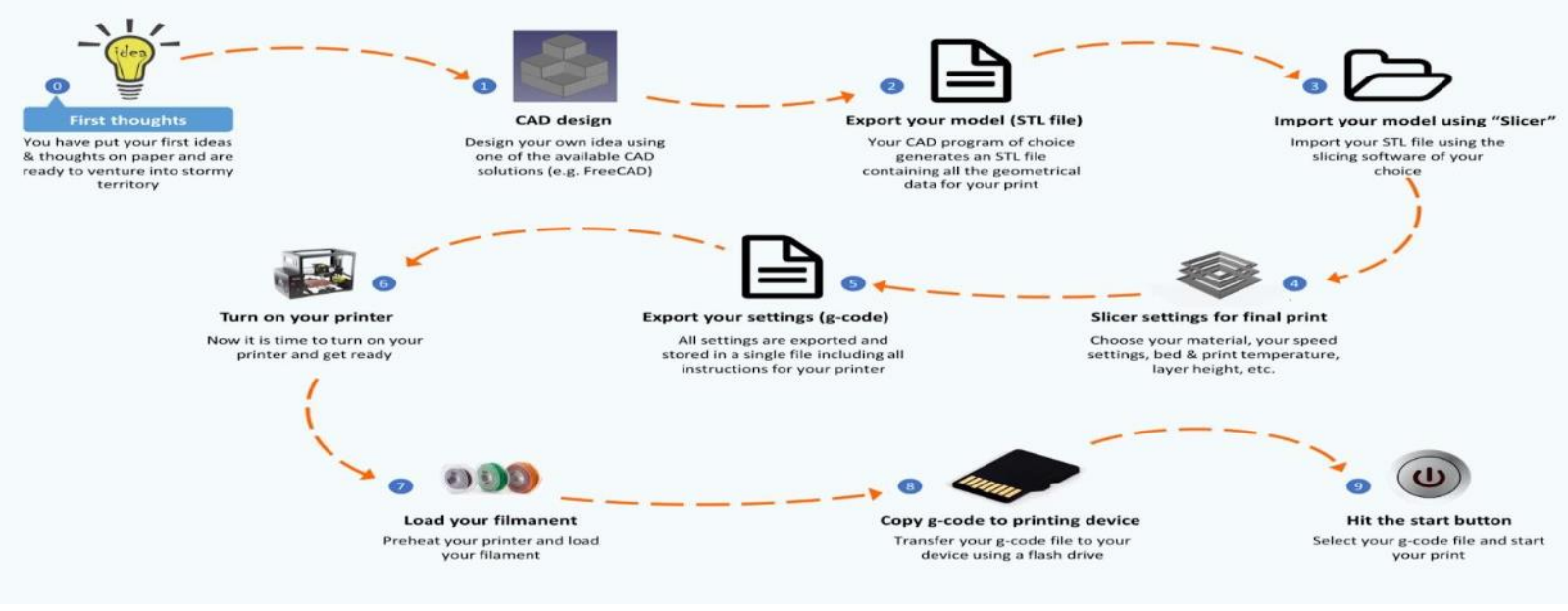

Fig..8. 3D Printing Process flow chart

\subsubsection{CAD Model}

The first basic requirement of any 3D printing may be a CAD Model. It's the 3D design for the merchandise you would like to print. This model is often developed from various software's (Catia, Fusion360, SolidWorks, Creo, etc.) but the ultimate output has got to be during a computer readable format, mainly STEP, STL \& OBJ but a couple of other formats also are used[19]

\subsubsection{Slicing}

The designed model is now to be loaded into slicing software. The slicing software or Slicer, literally slices the 3D model into multiple layers counting on the specifications you provide. These slices (also called as layers) are then deposited one above the opposite during the particular printing. The slicer converts the planning into coordinates which the printer understands and therefore the material is deposited as per the coordinates. The output of this slicer is within the sort of a document with a file extension being ' $\mathrm{g}$ codes' 20$]$ 


\subsection{3. fixing the Machine}

The parts are often printed through various 3D printing technologies and counting on the ultimate application of the part, the acceptable technology \& material is chosen and the machine is about up. FDM printers use filaments like PLA, ABS, PC, PET-G, etc. while SLA \& DLP printers use resins with usagebased properties (tough, flexible, dental etc.) \& SLS uses powdered material (mostly Nylon)[21]

\subsubsection{D Printing}

The next step is to easily 3D prints the model. The gcode file is loaded into the printer and therefore the printing starts. The printer will print the thing as per the print parameters set within the slicer. These settings are often modified for each single print. The printing time depends on various factors and may vary from minutes to hours to even days[22]

\subsection{Processes for biomedical 3D printing}

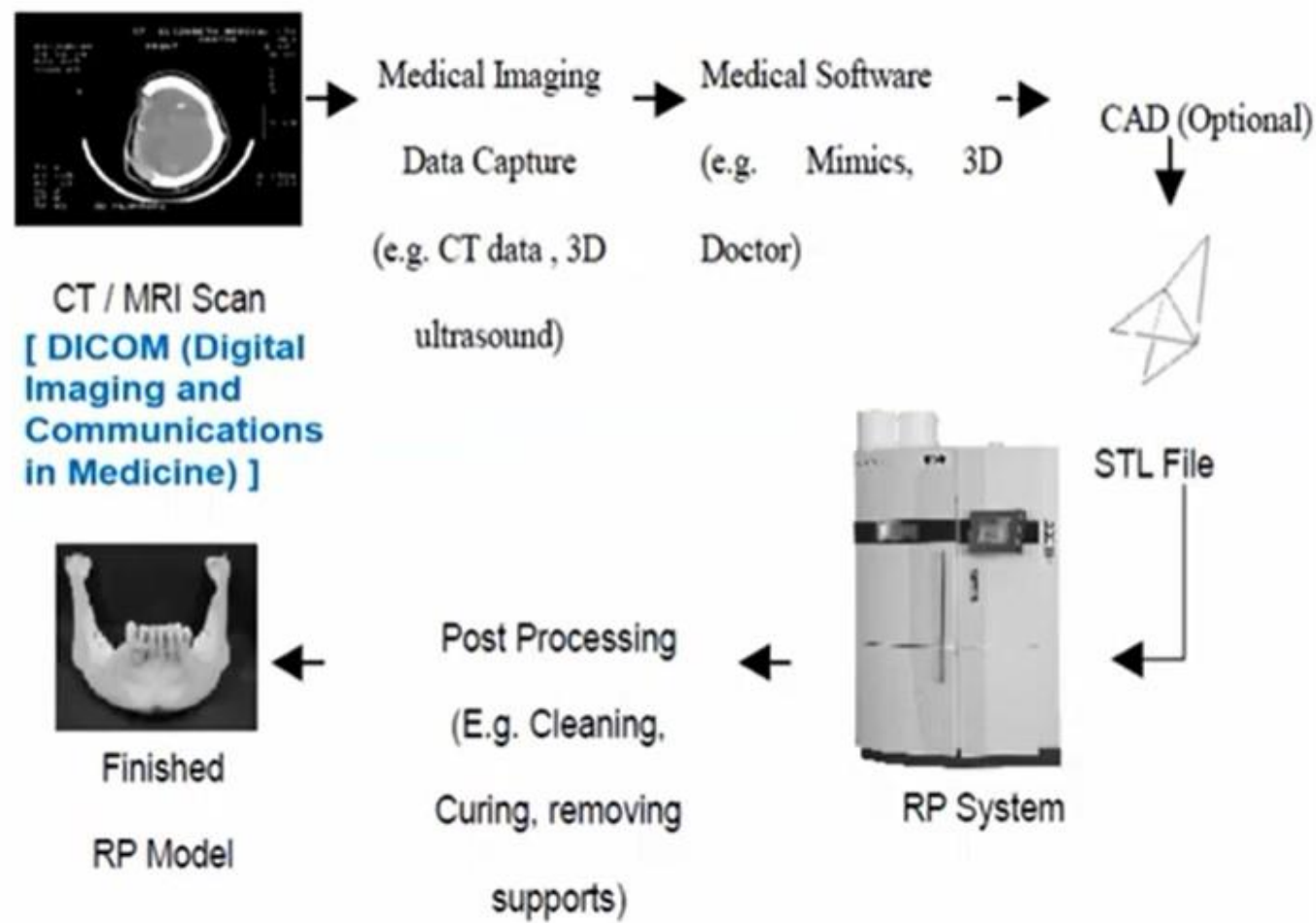

Fig.9. Processes for biomedical 3D printing 


\subsubsection{Medical Imaging:}

we imported sub-millimetre Digital Imaging and Communications in Medicine (DICOM) CT data into Mimics Medical 20.0 software (Materialise, Leuven, Belgium). Manual bone threshold segmentation was performed to create a 3-D virtual model of the patient's chest. This was digitally reconstructed in 3-D from the sub millimetre CT slices[23] As the CT scanner data varies from case to case, we used manual adjustment by eye, which has previously helped us provide best results over automatic segmentation and region growing, and was used. The ribs were clearly visible on CT slices to produce the Stereolithographic (STL) files. The STL files of the ribs, the tumour and sternum were imported into Geomatic Freeform Plus Software An anatomical image of the ribs and right hemi-sternum to be reconstructed were then digitally obtained from the chest CT scan and the proposed titanium implant, as a single part in titanium alloy, was fashioned to mimic the shape and contours of the ribs and sternum[24]
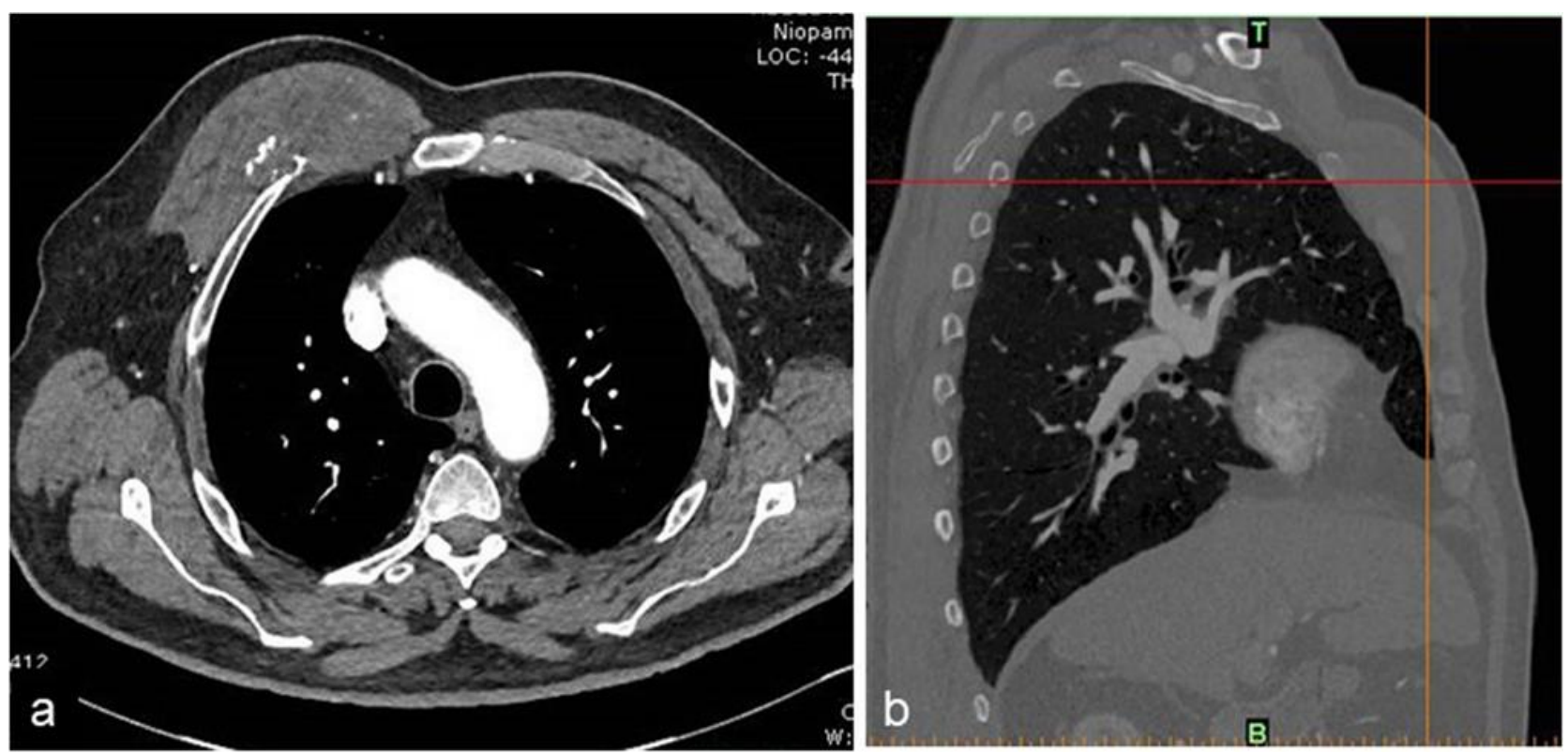

Fig.10. Medical imaging(DICOM file)

\subsubsection{Software's used for slicing:}

Icrease in the availability of open-source software and 3D designs for printing. Many of these freely available tools are translatable alongside new ones that are directly applicable to the biomedical field. A few examples are detailed here[25]

\subsubsection{3D printers}

- REPRAP - an online resource for all things 3D printing, including how to build custom 3D printers. As many bioprinters have unique applications and require specific functions, many groups have modified low-cost FDM printers for affordable bioprinting set-ups.

- Feinberg et al this group recently released a complete set of instructions along with .stl files to print a "large volume syringe pump extruder for desktop 3D printers". This enables the user to convert a low-cost FDM filament printer into a bioprinter for the extrusion of gel or paste-like bio inks[26] 


\subsubsection{3D CAD models}

- NIH 3D Print Exchange an online free database of scientifically accurate and medically applicable models. Ranging from prosthetics to protein structures, the database has almost 7000 3D models that are free to download.

- Thing verse (MakerBot) - a large online database of general 3D models. It includes a selection of anatomical models and models for a range of medical tools and devices uploaded by the printing community[27]

\subsubsection{Slicing software}

- Slic3r, Cure (Ultimaker), Repeater-Host, etc... there are many different open-source slicing packages available that all have similar functions and can be adapted for use with most 3D printers[28]

- Petri Printer - a G-code generator developed with bioprinting for cell culture in mind, this software enables the user to design printing set-ups for multiple culture plates or petri dishes.

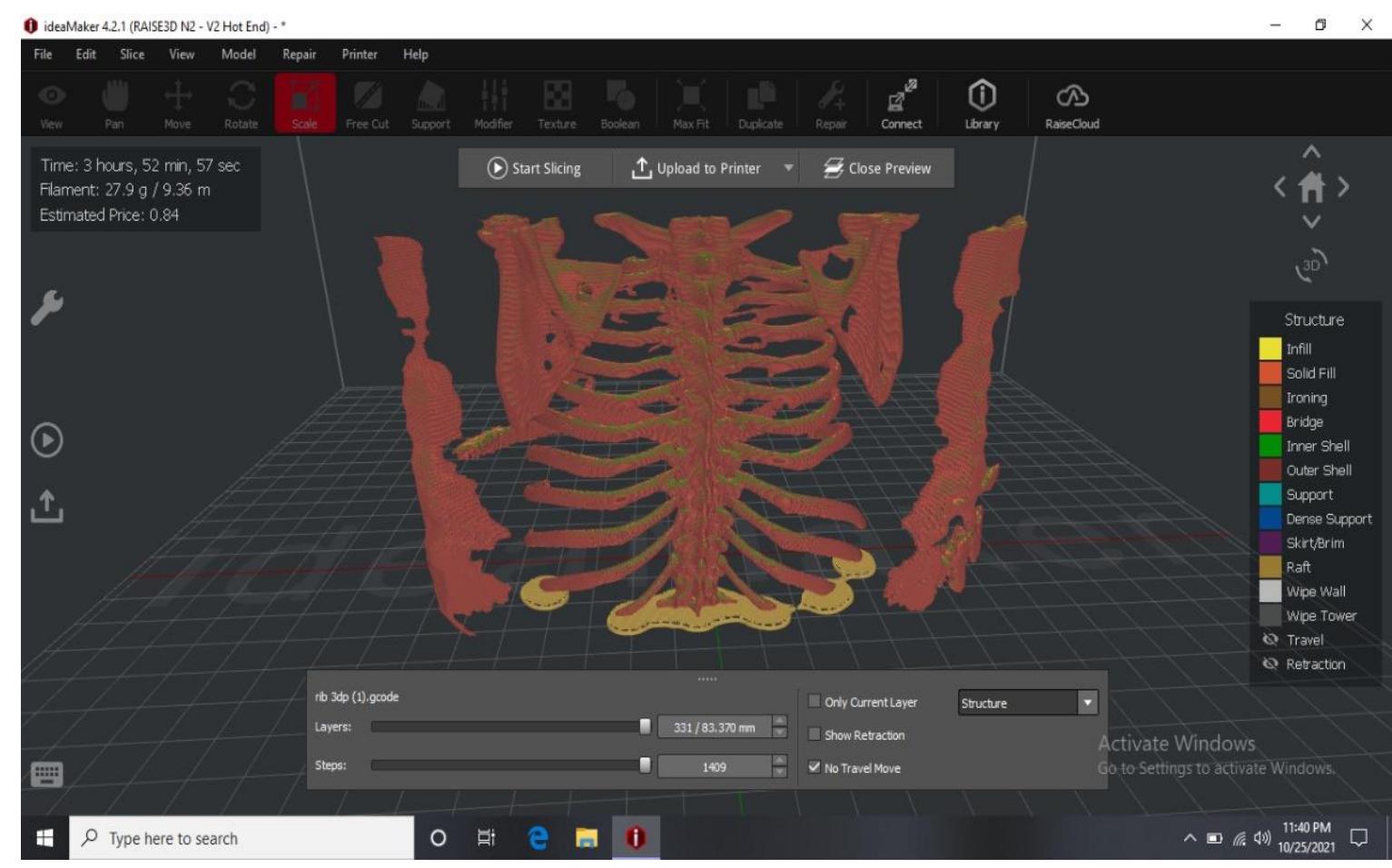

Fig.11. Slicing software

\subsubsection{Resources}

- Bio verse (Celink) - an online community of researchers involved in bioprinting where CAD models, protocols and other resources are shared for free.

- Embodi3D - an online biomedical 3D-printing community providing printable anatomic body parts (.stl files) and tools for converting medical DICOM image stacks into printable files as a freeware service[29] 


\subsubsection{Post Processing:}

This important step of the 3D printing process is known as post-processing. Post-processing in 3D printing refers to any process or task that needs to be performed on a printed part, or any technique used to further enhance the object. Think of it as a finishing touch to treat and refine parts that come out of a 3D printer. The options for post-processing 3D printed parts include removing support or excess material, washing and curing, sanding or polishing a model to painting or colouring[30] We can identify 5 steps in post-processing, although not all steps are required for all projects:
1. Cleaning
2. Fixing
3. Curing or hardening
4. Surface finishing
5. Colouring

Also, beware that the post-processing technique can vary depending on the printing process used to create the model.

\subsubsection{Cleaning}

a) Removing support material (FDM and Material Jetting) When printing models with overhang on FDM or other material jetting technologies, support structures that hold up the overhanging features are needed. These support structures can be printed using the same material as the one with which the model itself is printed. But when the machine allows for printing with multiple materials, special support material can be used. Nonetheless, every time a support structure is required, there will be some post-processing involved. There are two types of support material: soluble and insoluble (usually the latter is the same material the model is printed with). Insoluble material is relatively strong and can only be removed using tools as knives or pliers. This has to be done carefully and there exists the risk of damaging the model, or inadvertently removing small features. When using soluble support material, there is a lower risk of damaging the model[31] The support structures can be dissolved in water or with a chemical called Limonene. Examples of soluble materials are HIPS (used as a support with ABS material) and PVA (used as a support with PLA material)

b) Powder removal (SLS and Powder Bed Fusion) Models printed using powder bed fusion (SLS, etc) are fabricated using plastic or metal powders. Residues of powder can cling to or remain in the model, for example in holes or more complex internal channels inside the model. Excess powder can be removed manually but automated solutions have arrived on the market that vibrate or rotate to remove access powder. This 3D printing post-processing technique works like some sort of a centrifuge rotating the part in all 3 dimensions[32]

c) Washing (SLA and Photo polymerisation) Parts that are printed with SLA or other photo polymerisation can be easily cleaned after printing. Two companies added post-processing washing machines that are seamlessly integrated in their print process line up. Form Labs added the proper wash which uses isopropyl alcohol (IPA) to clean the parts. Carbon developed the Smart Part Washers machine to do the cleaning[33] 
1.7.3.2. FixingSometimes small repairs are needed to fill small holes or cracks or even to attach together parts that have been printed separately.

a) Filling When fillers and hardeners are used to repair unwanted holes or cracks in the printed object.

b) Gluing and welding Used when separately printed parts need to be attached together. ABS prints can be welded or glued together using acetone.

1.7.3.3. Curing Just like French fries, baking the models after they have been printed enhances the mechanical properties (crunchiness in case of the fries) of the material. Form labs and Carbon have added curing using UV light to their printing process (SLA and CLIP respectively, both Photo polymerisation processes[34] After the model has been printed special curing machines heat the model to bring the part to its optimal mechanical properties. Curing therefore differs from the other post processing options, that it enhances not just the aesthetic characteristics, but the physical quality of the model.

\subsubsection{Surface Finishing}

After the washing, cleaning, removing support or excess material and curing, different processes are available to make the model look nicer aesthetically. This is especially relevant when the models are geared towards consumer markets[35]

\section{a) Sanding}

Layer lines or touch-points where support structure was attached to the model can be removed by carefully sanding the surface of the model, using sanding paper with varying grit from to from low too high for finishing. Aside from being labour intensive, manual sanding can create inconsistent results. With automated polishing, this can be avoided. Layer lines are particularly visible on 3D models produced using layering techniques (like FDM)[36]

b) Vapour or Chemical Smoothing. Sometimes chemicals are used to smoothen the model surface. The vapours react with the outer layer of the object. The layer lines are melted away, leaving a smooth outer layer while giving the model a glossy look. For models printed with PLA and ABS the acetone is often used, or the chemical agent Tetrahydrofuran (THF). The problem with this technique is that is cannot be controlled: small features can be melted off that should remain. Also, the vapours can be harmful when inhaled. This can be avoided using closed chemical cleaning machines[37]

\subsubsection{Colouring}

In some cases, 3D models can be printed using coloured material and with multi-material printing (multi-) coloured prints can be made. But one can also opt for colouring during the post-processing phase[38]

- Coating and painting Parts that need colouring would ideally be printed using white material. Before the model is painted a layer of primer is usually applied. Painting can be done manually using a brush or spray. There are machines that automate spraying of parts. 


\section{LITERATURE_SURVEY}

Muhammad Usman in his research paper he explained about the general procedure that need to be follow in general printing of 3D objects in 3D printing technology like designing, modelling, and slicing. He also explained software's the detailed specifications of the end products that were printed using the Delta 3D printer used in the 3D Printing Technology[39]

Ying-Yi Chen ,Kuan-Hsun Lin ,Hsu-Kai Huang ,Hung Chang, Shih-Chun Lee, Tsai-Wang Huangin their article provided the beneficial beneficial application of three-dimensional (3D) printing for surgical stabilization of rib fractured patients and they study about the behaviour of the patients after the surgery[40]

Prof. Alfonso Fiorelli in his article he explained about the Preoperative Planning for implementation of $3 \mathrm{D}$ body part that need to be implemented in the surgical treatment of chest wall disease and Four studies reported comparison with a conventionally treated control group, and Satisfactory morphological correction was reported in all studies, and six studies reported a good implant fitting with minimal need for intraoperative adjustments of implant body part. There were no major intraoperative or postoperative complications in any of the studies[41]

Prof. Yahya Bozkurt and Elif Karaye. In this study, explained about the 3D printer technology is introduction, various method of 3D printing is mentioned and the use of this technology in biomedical applications is referred. The use of 3D printing in surgery, pharmaceutical industry, disease modelling, development of customized implants and prostheses, organ printing, vet medicine and tissue engineering applications have been explained and this new method compared with traditional methods that used in the biomedical field. In addition, this study includes future opportunities that are expected to become widespread and developed in the future[42]

Tuan D. Ngo, Alireza Kashani, Gabriele Imbalzano, Kate T.Q. Nguyen, David Hui this article discussed the main processing challenges with void formation, anisotropic behaviour, the limitation of computer design and layer-by-layer appearance and discussed about the biomaterial used in the biomedical field A comprehensive review of the main 3D printing methods, materials and their development in trending applications was carried out. In particular, the revolutionary applications of AM in biomedical, aerospace, buildings and protective structures were discussed[43]

Carlota Vin this the rib implant was 3D printed in titanium, a material that is a biocompatible. During the operation, the surgeon Ehab Bishay replaced the portion of the patient's damaged rib cage with the 3D printed titanium device to add support. Linda Edwards was only the sixth person in the world to have this type of surgery[44] 
He made an study on a 52-year-old, patient in Linda Edwards successfully who has successfully received a $3 \mathrm{D}$ printed cage implemented about the process and his health condition after the surgery. The surgery took place in Birmingham, at Queen Elizabeth Hospital.

Xue-tao Zhou, Dong-sheng Zhang*, Yang Yang, Guo-liang Zhang, Ze-xin Xie, Meng-hui Chen and Zheng Liang in their journal of cardiothoracic surgery Retrospectively they have analysis of 5 clinical cases of multiple rib fractures from January 2017 to August 2018 in our hospital. A preoperative CT thin slice scan was used to reconstruct the $3 \mathrm{D}$ model according to the scanning results, and $3 \mathrm{D}$ printing technology was adopted to prepare the rib mode. They made the rib model and the pre-shaped titanium alloy rib locking plate using 3D printing technology, provided a more minimally invasive and precisely individualized treatment for some rib fracture operations[45]

L.-C. Zhang, H. Attar1, M. Calin and J. Eckert in their article they briefly evaluates about the work carried out on the significance of Titanium materials, selective laser melting(SLM) technology and selective laser melting(SLM) manufacturing of Titanium materials commonly used for biomedical applications including the micro structures and mechanical properties of resulting bulk dense parts ( $\mathrm{Ti}, \mathrm{Ti}-$ $24 \mathrm{Nb}-4 \mathrm{Zr}-8 \mathrm{Sn}, \mathrm{Ti}-6 \mathrm{Al}-4 \mathrm{~V}, \mathrm{Ti}-6 \mathrm{Al}-7 \mathrm{Nb}, \mathrm{Ti}-\mathrm{TiC}$ and $\mathrm{Ti}-\mathrm{TiB})$ as well as the porous structures successfully produced by selective laser melting(SLM[46]

Aneez D.B. Ahmed, Prajwala S. Prakash, Chia Ming Li Cynthia in their surgical medical report which shows the case study of the 2 old patients who are suffering from the chest wall cancer and they need to fix with some steel alloys and in the second case need to fix by titanium ally plates to with stand of ribs pressure[47] 


\section{METHODOLOGY}

Methodology :

problem statement

extracting the medical image (DICOM)

modelling the RIbcage in 3D slicer

curing the model in meshmixer

Adjusting the size and scale

Final output 


\section{MATERIALS}

\subsection{Titanium Alloys:}

Titanium alloys have been used for a long time to treat the trauma bone fractures. Titanium alloys have been found to be a good replacement for SS due to their better corrosion_resistance, less stiffness, and easier machining capabilities. However, Young's modulus of titanium alloy (113 GPa) is still much higher than that of cortical bone (15-20 GPa). Successful treatment of trauma bone fractures has been extensively published with good clinical outcomes using titanium alloy implants. Titanium alloy implants would provide bone-implant fixation with good strength under human physiological loading conditions by which the bone can be healed with high rate of fracture union. However, the quality of healed bone and union time are might not be effectively achieved in low mineral density bone. Even in young patients with good mineral density bone, the patient's functional needs might not be entirely obtained. Stiffness of bone-implant construct for early callus formation, stress shielding at the final stage of bone healing, fixation of screws to the bone, loss of reduction, fixation misalignment, implant removal, etc., are some clinical challenges that have risk of failure and cause unsuccessful clinical outcomes. Less pain and natural joint function at the fracture_site are the final clinical outcomes that are desired by the patients.

The successful outcomes of titanium alloy implants have been reported in a number of papers for distal radius plate, distal femur plate, proximal_tibia plate, one third tabular plate, reconstruction plate, distal humerus plate, proximal humerus plate, and hook plate for olecranon. Despite successful reported outcomes for the titanium alloy implants, several biomaterials (e.g., PEEK composites and biodegradable magnesium) have been found to be promising for use in trauma implants. Although the mechanical side effects of titanium alloy could limit the effective treatment of the trauma bone fractures, it is still trustable material for use in trauma implants. The mechanical properties of this material could be further developed to reduce the mismatch with cortical bone. However, the treatment of osteoporosis bone is the key for development of trauma implants in view of material, implant structure, and mechanism. In bones with low mineral density, bone grafting has been utilized to enhance the bone healing in conjunction with titanium implants. Titanium implant may introduce soft tissue irritation or rupture (e.g., flexor_tendon rupture in distal radius zone), which necessitates removal of the plate.

Higher biocompatibility, higher corrosion resistance, and lower mismatching modulus with cortical bone have been contemplated for development of biomedical titanium alloys. Although the Ti6Al4V has good mechanical strength and corrosion resistance, the release of vanadium (V) and aluminium (Al) ions may limit the usability of this material for long term. Reduction of $\alpha$ phase in titanium alloys diminishes Young's modulus; therefore, the titanium alloys with higher $\beta$ phase ( $\beta$ titanium) have been explored for use in trauma implants. Titanium alloys with high percentage of $\beta$ phase (e.g., Ti-35Nb-7Zr-5Ta, Ti29Nb-4.6Zr-13Ta, Ti-15Mo (TM), and Ti-15Mo-5Zr-3Al (TMZA)) have higher corrosion resistance and better ductility compared to $\alpha+\beta$ titanium alloys (Ti-GAl-7Nb (TAN), Ti-6Al-4V (TAV), and Ti-6Al-4V ELI (TAV ELI)). It was found that by microstructural engineering of titanium alloys, optimum microstructure phases of $\alpha+\beta$ could be developed to achieve titanium alloys with lower Young's modulus and good mechanical strength for use in trauma plating systems

\subsection{Nickel-free austenitic stainless steels}

Corrosion-resistant nickel-free austenitic stainless steel can be produced if high amounts of chromium, molybdenum and nitrogen are alloyed in the steel. Both nitrogen and carbon are potent and effective stabilizers of austenite structure; however, small addition of carbon can decrease the corrosion resistance 
of steel and also enhance its tendency to form precipitates. Therefore, the carbon content in medical stainless steel is restricted to less than $0.03 \%$. The best and only acceptable element to stabilize the austenitic phase in nickel-free steels should be nitrogen. Thus, nickel-free austenitic stainless steels have been developed in which nickel is completely replaced by nitrogen. Additionally, high nitrogen content is also responsible for the increased strength and improved corrosion resistance.

High-nitrogen stainless steels, in addition to high strength, also show high work-hardening rates and good high-temperature mechanical properties as compared with conventional steels. The metallic implants which replace the failed hard tissues, such as artificial joints, bone plates and dental implants, are conventionally used under severe cyclic loading conditions. Therefore, the high-nitrogen nickel-free stainless steels, which typically exhibit high strength, ductility and toughness, are the perfect candidates for the structural material of these implants.High-nitrogen nickel-free stainless steels with lower toxicity to human body constitute the next generation of stainless steels for surgical implant applications. The currently used metallic implants still suffer from the problem that their elastic modulus is different from that of the bones. However, mechanical properties of the porous metals can be adjusted to match those of replaced bones by changing the porosity and pore sizes. The pores on metals can also permit good attachment of tissues to the surface of biocompatible metals, allowing the tissue to enter the metals.

In the past decade, several high-nitrogen nickel-free austenitic stainless steels have been developed for medical application, mainly focusing on $\mathrm{Fe}-\mathrm{Cr}-\mathrm{N}, \mathrm{Fe}-\mathrm{Cr}-\mathrm{Mo}-\mathrm{N}$ and $\mathrm{Fe}-\mathrm{Cr}-\mathrm{Mn}-\mathrm{Mo}-\mathrm{N}$ systems. Nickelfree stainless steels generally show excellent mechanical properties and even in the annealed condition (soft state), their strength is much higher than that of the conventional stainless steels used for implants, e.g., $316 \mathrm{~L}$, whereas their elongation is comparable. As the strength of stainless steels can be increased by cold deformation, nickel-free stainless steels also exhibit a stronger potential for work hardening than the conventional stainless steels. This will open up new possibilities for higher strength implants or for reduction of implant sizes where limited anatomical space is often an issue, for instance, microvascular stents with finer meshes. Although the evaluation of nickel-free stainless steels with regard to surgical implants is yet incomplete, these steels demonstrate satisfactory biocompatibility and might be used in medical fields in the near future. 


\section{DESIGN AND DEVELOPMENT OF RIBCAGE}

\section{1 . EXTRACTING THE DICOM FILE}

This is the initial stage of the ribcage modelling process that is to extracting a DICOM file (Digital imaging and Communications in medicine) from a patient who need to implant the ribs it provides the all-directional view to understand the patient condition. DICOM is the international standard to communicate and manage medical images and data. Its mission is to ensure the interoperability of systems used to produce, store, share, display, send, query, process, retrieve and print medical images, as well as to manage related workflows[48]

\subsection{DESIGN THE RIBCAGE IN 3D SLICER}

\section{The technology behind a medical imaging system}

Healthcare organizations are gradually moving toward a population health management model and better patient enablement, and medical imaging informatics is at the forefront. Here's a breakdown of the major technologies that store and share images and allow them to be viewed.

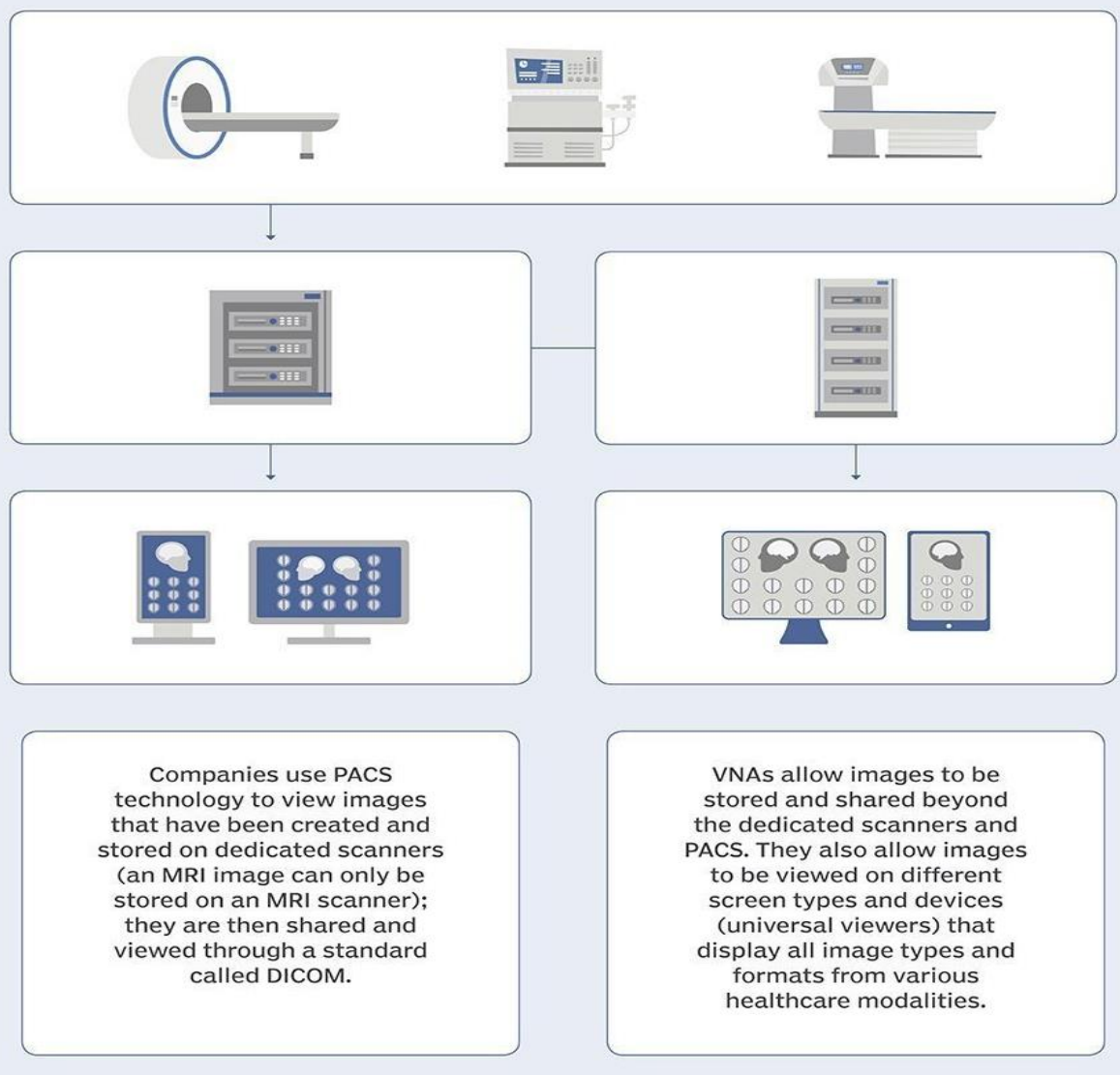

Fig.12. Extraction of DICOM file 
The slicer acts as the middleman between the 3D model and the 3D printer. Once you have modelled the object you would like to 3D print, you will have it in an STL file. In this we use 2 main slicing software tools to obtain the full 3D ribcage in the format of STL file (Standard Tessellation Language). The main two tools are

\subsubsection{VOLUME RENDERING}

Volume rendering is a type of data visualization technique which creates a three-dimensional representation of data. CT and MRI data are frequently visualized with volume rendering in addition to other reconstructions and slices. In this we need to adjust the volume scaling, nodes and vertices count. Mostly we specify the volume scale to adjust the size of the STL file and to get a perfect size object. Set the present in the MR-Default to obtain the output[49]

\subsubsection{GRAYSCALE MODEL MAKER}

In this tool we adjust the volume properties and naming of the model also we add smoothening surface to the volume of the ribcage after adjusting the all properties and apply the adjustments it converts the design into a single $3 \mathrm{D}$ object of ribcage in this process, we need to adjust the volume according to the patient measurements and size and thickness of the bone. Now we can save the file after completing the two tasks we need to save the file in STL format.

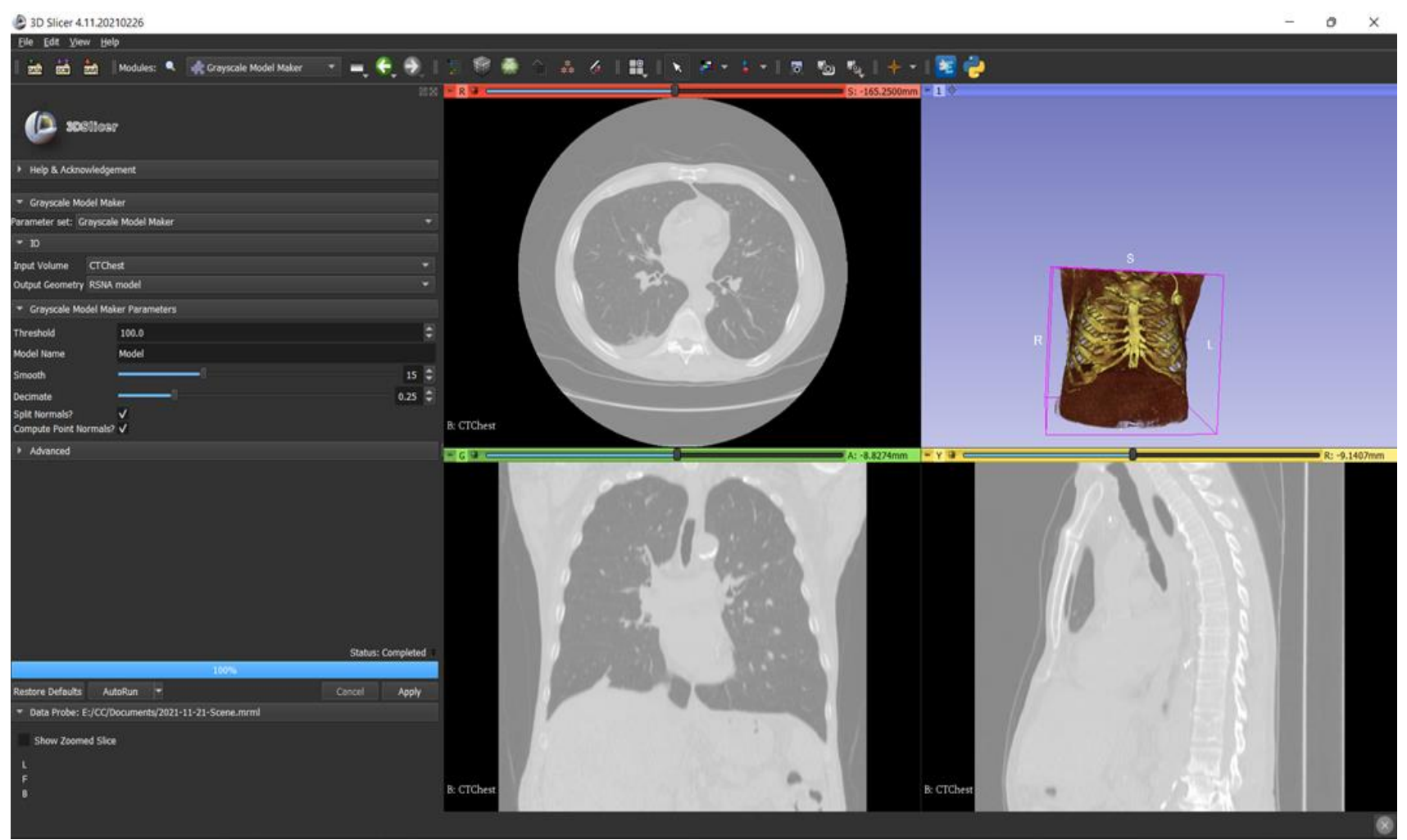

Fig.13. GRAYSCALE MODEL MAKER 


\subsection{DESIGN IN AUTODESK MESHMIXER}

Autodesk meshmixer is a fantastic free software for creating and manipulating 3D files for 3D printing. Whether you need to clean up a 3D scan, do some 3D printing or design an object, meshmixer can help. In this meshmixer software we perform a lot of tools to get final 3D output, from those the main some types are there they are[50]

\subsubsection{SELECT}

The STL file we got from the 3D slicer contains lot of blood and muscle particle attached to the ribcage those blood and muscle particles are removed by this tool the unwanted parts need to be selected and use the discard tool command to eliminate are remove the unwanted particles by the help of other sub tools present in the select tool[51]

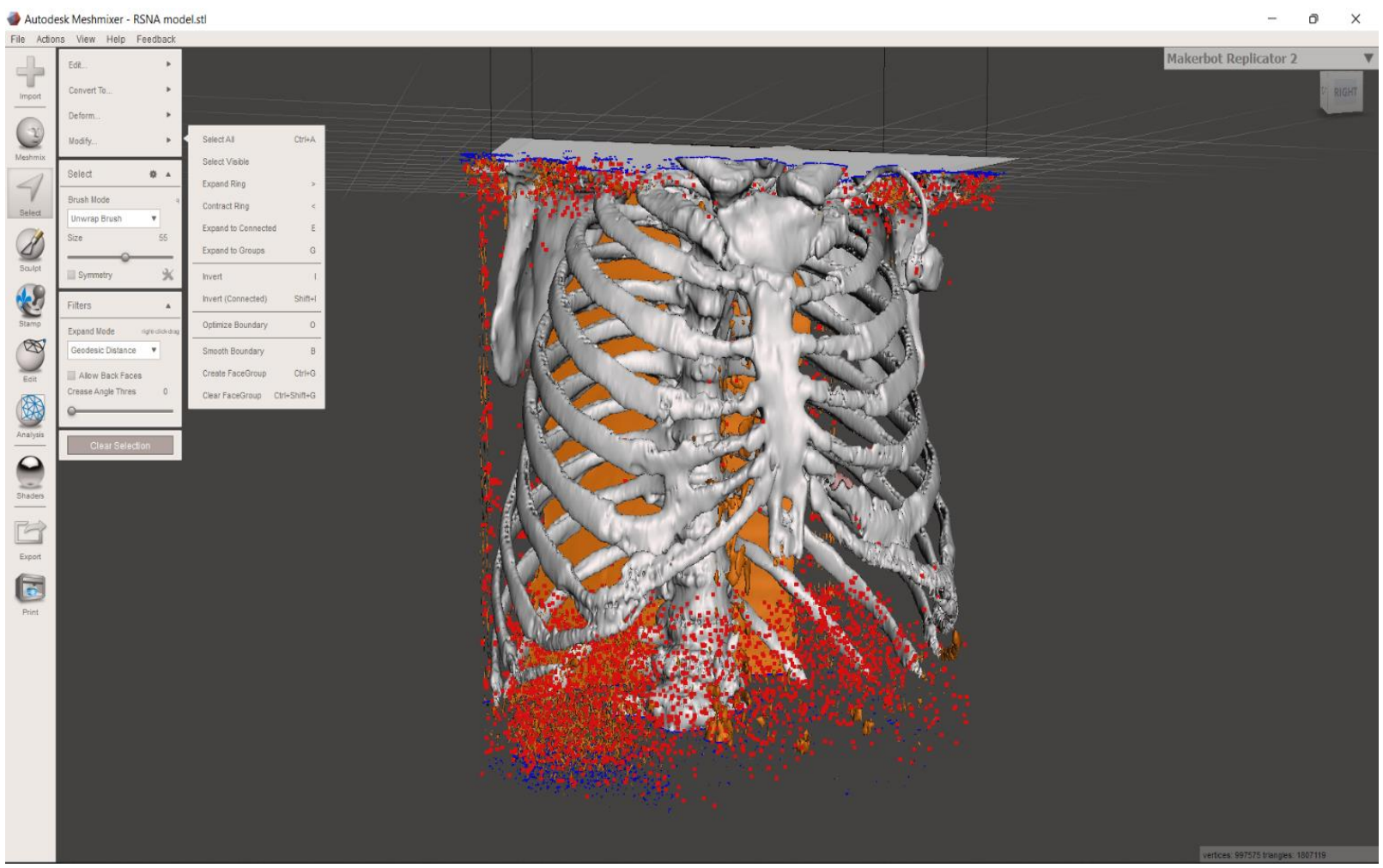

Fig.14. Removing of unwanted material.

\subsubsection{ANALYSIS}

In this analysis tooling step, we need to find the errors in the file through the sub tools called as inspection because due to the errors we can't get a desired output. The 3D printer did not know how to deal with the errors in the printing time it leaves the thing empty which may cause defects.Those errors can be corrected through the auto repairing process it corrects the most of the errors in a single time it is the best and easy way to reduce the errors in the output of ribcage[52] 


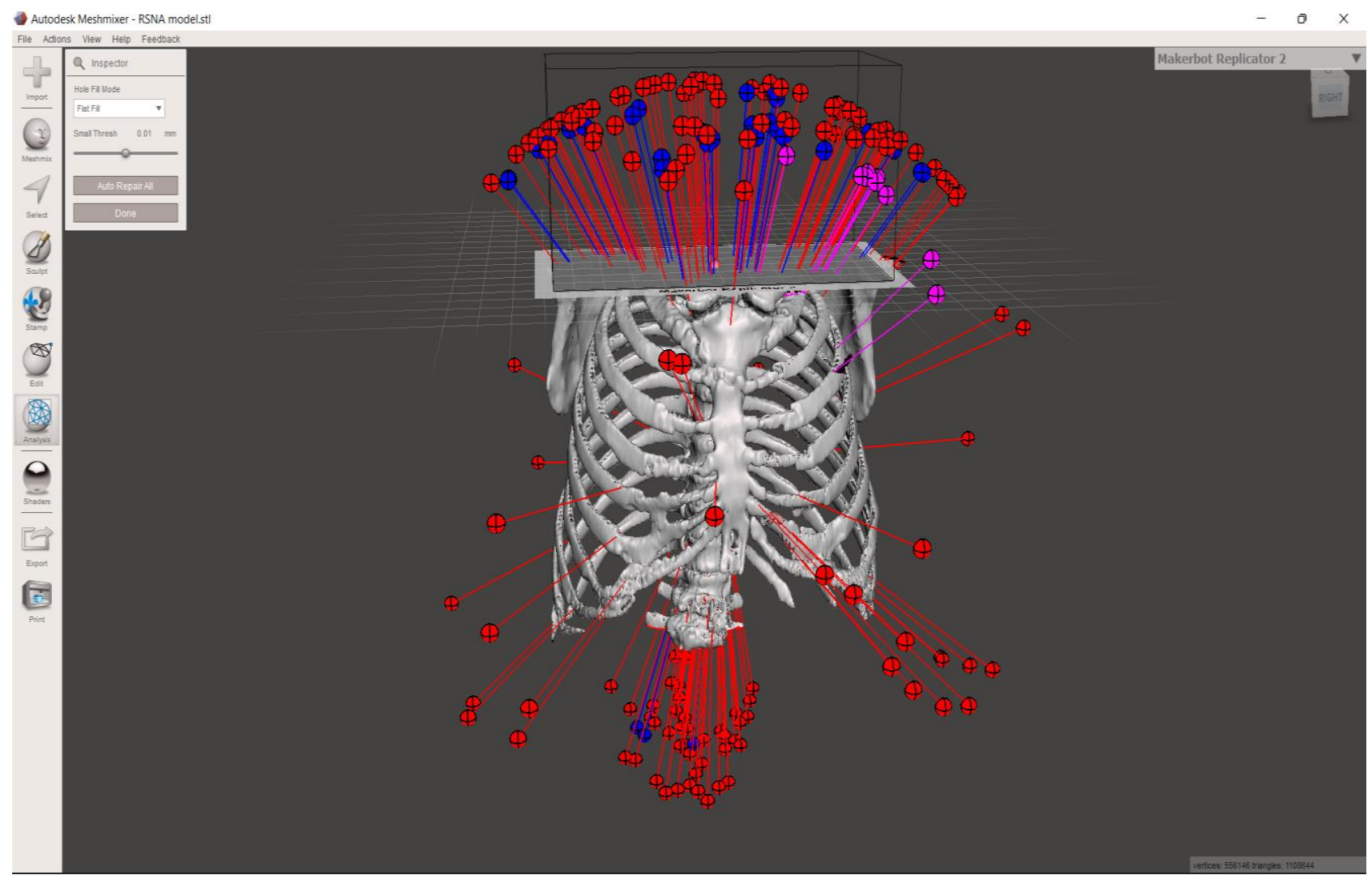

Fig.15. Analysis the erro

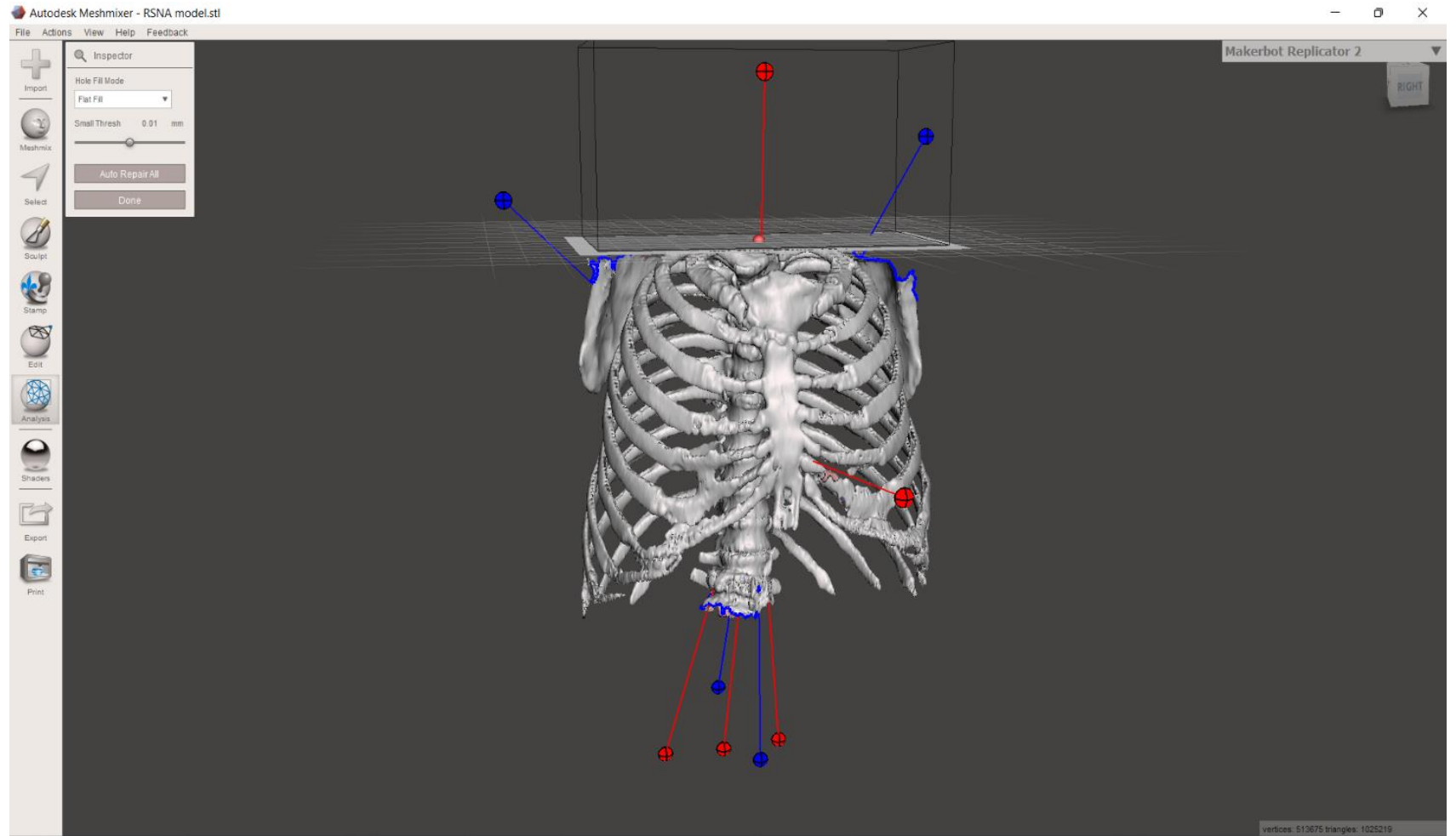

Fig.16. Rectifying the errors 


\subsubsection{RE-MESH}

This is the sub tool present in the select tool which is used to smoothen the surface of the object .It attempts to replace the entire the surface with new polygon and new triangles that are about same size and same density it redo the entire mode keep the same geometry of the ribcage[53]

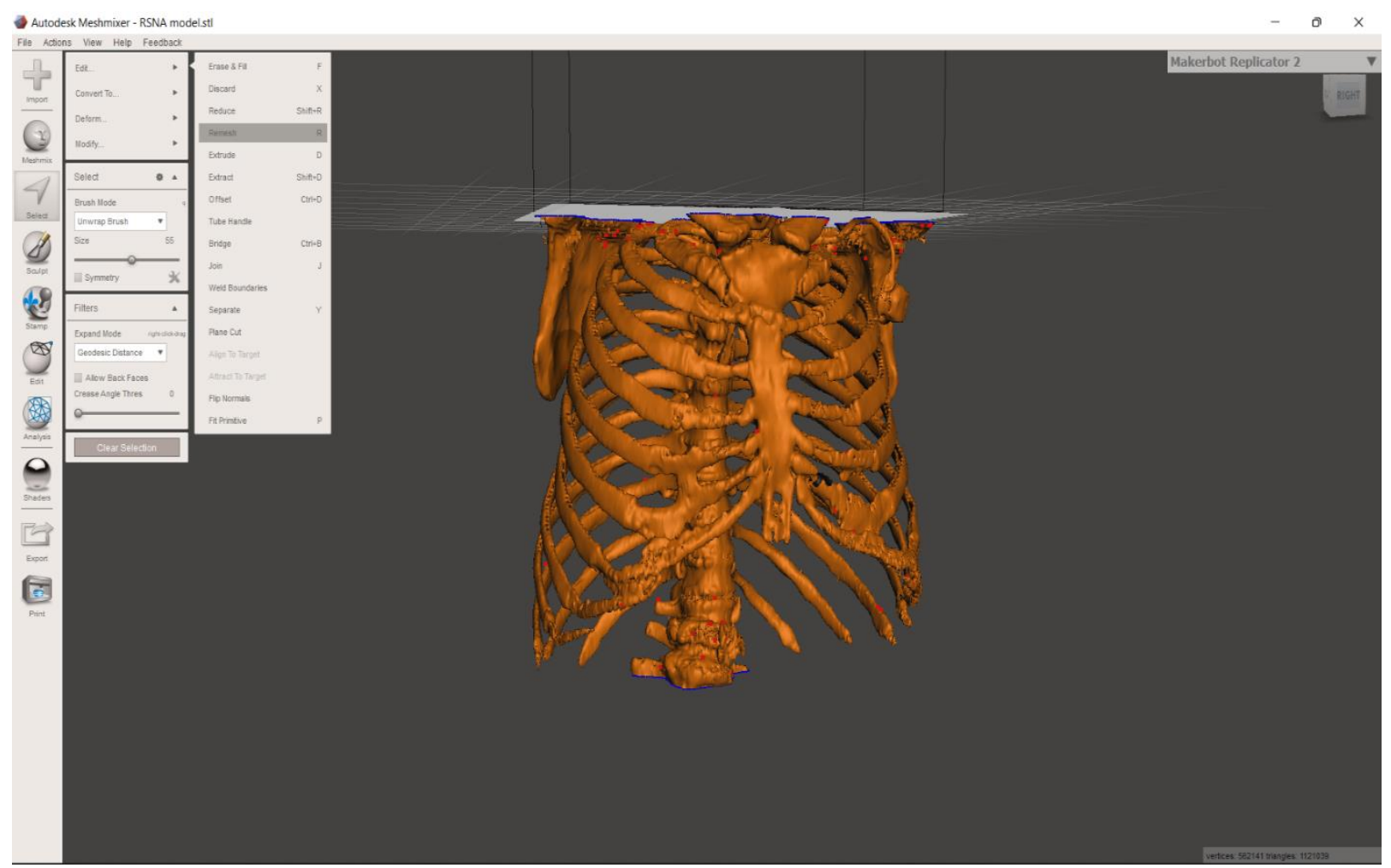

Fig.17. Re-meshing 


\section{Problem statement}

To develop a Ribcage prototype, it is essential to generate a model design of ribcage which is able to resist the temperature of the body and reaction free. Prosthesis these days have become a common entity in need for many amputees suffering especially who had a chest tumour and rib damaged patients in accidents. In spite of expensive and long duration process this 3D printing technology give their best functionality for optimal requirements. For these reasons we choose 3D printing Ribcage. Our main aim is to provide an inexpensive and long-life ribcage .

\section{Objectives}

The various objectives of this project are listed below:

To design an inexpensive designing process of prosthetic ribcage .

To create a non-reactive object with the blood and muscle .

To design a temperature resistance ribcage to withstand the internal body temperature.

\section{RESULT}

Metals are by far the most traditional surgical materials. Because of crucial and detrimental effects of metal corrosion on human body, the history of implant metals development has been largely focused on seeking better corrosion-resistant materials. Today, the widely used metallic biomaterials mainly include stainless steels, titanium and its alloys, cobalt-chromium-based alloys, as well as tantalum, niobium and gold.

The first stainless steel used for implants contains $\sim 18 \mathrm{wt} \% \mathrm{Cr}$ and $\sim 8 \mathrm{wt} \%$ Ni makes it stronger than the steel and more resistant to corrosion. Further addition of molybdenum (Mo) has improved its corrosion resistance, known as type 316 stainless steel. Afterwards, the carbon (C) content has been reduced from 0.08 to $0.03 \mathrm{wt} \%$ which improved its corrosion resistance to chloride solution, and named as 316L.

Stainless steels, AISI 316L as a representative, are conventionally used in orthopedics, with main advantages of low cost, good mechanical properties and easy processing. However, problems were found with medical stainless steels during the last decades of clinical applications. Firstly, the medical stainless steels are denser, stronger and have higher elastic modulus than bones, so this incompatibility of strength or modulus can cause the shielding effect of stress and worsen the bone healing processes. Secondly, there is inevitable corrosion and wear of the medical stainless steels in body fluid environment, such as crevice corrosion, intergranular corrosion, pitting corrosion and fretting corrosion. These factors probably lead to an early fracture or failure of the implants, and corrosion of the implanted devices may result in release of harmful products into the body. Finally, the most important problem is the negative effect of metal ions or fretting debris, which can be released from the stainless steel implant 
devices because of corrosion, wear or other reasons. Nickel and chromium are known as potentially harmful elements in the medical stainless steel. Nickel ions act as the allergens, which may cause cutaneous inflammations such as swelling, reddening, eczema and itching on skins, and may also lead to allergy reactions, teratogenicity and carcinogenicity in human body.

Because of the harmful effect of nickel ions from the austenitic stainless steels, such as AISI 316L, to human body, the high-nitrogen nickel-free austenitic stainless steels, generally the $\mathrm{Fe}-\mathrm{Cr}-\mathrm{Mn}-\mathrm{Mo}-\mathrm{N}$ system, are thought as a potential replacement in recent years. Nitrogen is a strong austenite formation element and has been successfully used to replace nickel that largely improved the mechanical properties and corrosion resistance of steel. Considering the potentially detrimental effect of nickelcontaining stainless steels on the human body, experts suggested to forbid or restrict the nickel content in the medical stainless steel products, such as medical implants, dental materials, ornaments and so on. Therefore, the medical grade high-nitrogen nickel-free austenitic stainless steels are becoming an important medical metal material.

\section{DISCUSSION}

3D printing has been developed in many fields of biomedicine. They are investigated the applications of 3D printing for anatomy and surgical education and neurosurgery in the literature review of the PubMed and Web of Science databases. These applications significantly improved the quality of anatomy and surgical education and practices. 3D printing produced accurate simulations of patient-specific anatomy, which can be used for preoperative planning and skill acquisition and demonstrates advantages in cadaveric dissection, plastic models, and plastination of cadaver specimens.

We utilized 3D printing to create a personalized design model to assist in rib fixation surgery. our data showed that 3D printing for surgery significantly reduced the operation time and aided in preoperatively determining the surgical plans, such as the location and length of the incision. Therefore, the preoperative 3D printing approach has the following three advantages for Surgery of ribcage fracture : (1) Ease of locating the rib fracture site and predicting the incision length, (2) Shortened operation time, (3) Useful for explaining the steps of surgery of ribcage fracture to patients and families Not all rib fractures should be fixed, because stabilization of chest wall is the most important., Actually, time consuming of intraoperative customization of plates depends on how much fragments of ribs and measuring thickness and curvature of different sites of ribs.

This new technique is not suitable for emergency conditions because 3D printing is time-consuming (at least 5-6 hours). We used computed tomographic images to generate 3D printing model in all kinds of rib fractures, even in flail of chest or non-aligned rib fractures. The cost is an additional limitation. Rapid prototyping machines cost $\$ 100$ to $\$ 200$ without including the cost of the plastic and resin-based materials. The developments of 3D printing will include different range of materials and lead to more durable and realistic products in the future by the advancement of technology. Berman [54] proposed that the cost and speed of 3D printers will improve, thereby increasing the usability and potentially the availability of these machines. Liman [55] said 3D printing will potentially revolutionize the anatomical and surgical sciences 
to the benefit of educators, surgeons, and patients in the future. Therefore, the 3D printing technique could become more valuable for rib fixation surgery. Prospective multi-institutional studies are needed to validate the feasibility and safety of 3D printing for the SSRF. Further clinical studies may provide more valuable results for patient outcomes.

\section{FUTURE SCOPE}

As of now in Biomedical field 3D Printing technology is used for tablets and prosthesis.

Used to print the artificial organs.

Tissue development.

\section{CONCLUSION}

Manufacturers want to decrease product development time, production time. Additive manufacturing could be one of the solutions. The sales of industrial grade 3D printing machines can build one-third the volume of industrial automation and robotic sales in 2022. It is foreseen that this figure could reach to $62 \%$ by 2025 . From the number of patents filed across the globe it is very much clear that a lot of development is taking place around this technology. Only 3520 patents were granted worldwide not counting duplicate filed in multiple countries in 2013. By 2018, the number is reached to approximately 22000 . The patents are related to additive manufacturing materials, software and equipment. Currently this technology is most widely used by aerospace and aviation industries followed by automobile, medical and footwear industries. With the passage of time, the technology could be affordable to masses. The day is not very far away when one can own 3D printer, design own stuffs, getting printed and flaunt off. The machine could also have the facility to recycle it.

Coming to the materials used in biomedical applications. The adverse effects of nickel ions release in human body have prompted the development of high-nitrogen nickel-free austenitic stainless steels for medical applications. In such steels, nitrogen not only replaces nickel for austenitic structure stability but also significantly improves steel properties. By combining the benefits of stable austenitic structure, high strength and good plasticity, better corrosion and wear resistance, and superior biocompatibility compared to the currently used 316L stainless steel, the newly developed high-nitrogen nickel-free stainless steel will be a reliable substitute to the conventional medical stainless steels. The potential applications of these novel steels include implantation materials, orthopaedic and orthodontic devices. 


\section{REFERENCE}

1. Campbell, Thomas, et al. "Could 3D printing change the world." Technologies, Potential, and Implications of Additive Manufacturing, Atlantic Council, Washington, DC 3 (2011).

2. Radenkovic, Dina, Atefeh Solouk, and Alexander Seifalian. "Personalized development of human organs using 3D printing technology." Medical hypotheses 87 (2016): 30-33.

3. Wang, Jie, et al. "Stereolithographic (SLA) 3D printing of oral modified-release dosage forms." International journal of pharmaceutics 503.1-2 (2016): 207-212

4. Kafle, Abishek, Eric Luis, Raman Silwal, Houwen Matthew Pan, Pratisthit Lal Shrestha, and Anil Kumar Bastola. "3D/4D Printing of polymers: Fused deposition modelling (FDM), selective laser sintering (SLS), and stereolithography (SLA)." Polymers 13, no. 18 (2021): 3101.

5. Long, Jingjunjiao, et al. "Application of fused deposition modelling (FDM) method of 3D printing in drug delivery." Current pharmaceutical design 23.3 (2017): 433-439.

6. Wittkopf, Jarrid A., et al. "3D Printed Electronics with Multi Jet Fusion." NIP \& Digital Fabrication Conference. Vol. 2019. No. 1. Society for Imaging Science and Technology, 2019.

7. O'Connor, H. J., Dickson, A. N., \& Dowling, D. P. (2018). Evaluation of the mechanical performance of polymer parts fabricated using a production scale multi jet fusion printing process. Additive Manufacturing, 22, 381-387.

8. Khaing, M. W., J. Y. H. Fuh, and L. Lu. "Direct metal laser sintering for rapid tooling: processing and characterisation of EOS parts." Journal of Materials Processing Technology 113.1-3 (2001): 269-272.

9. Bozkurt, Yahya, and Elif Karayel. "3D printing technology; methods, biomedical applications, future opportunities and trends." Journal of Materials Research and Technology 14 (2021): 14301450.

10. Bandyopadhyay, Amit, Susmita Bose, and Suman Das. "3D printing of biomaterials." MRS bulletin 40.2 (2015): 108-115.

11. Ngo, Tuan D., et al. "Additive manufacturing (3D printing): A review of materials, methods, applications and challenges." Composites Part B: Engineering 143 (2018): 172-196.

12. Han, Tao, et al. "3D printed sensors for biomedical applications: a review." Sensors 19.7 (2019): 1706.

13. Hwang, Henry H., et al. "3D-Printing of Functional Biomedical Microdevices via Light-and Extrusion-Based Approaches." Small methods 2.2 (2018): 1700277.

14. Wang, Xin, et al. "3D printing of polymer matrix composites: A review and prospective." Composites Part B: Engineering 110 (2017): 442-458.

15. Quan, Haoyuan, et al. "Photo-curing 3D printing technique and its challenges." Bioactive Materials 5.1 (2020): 110-115.

16. Wang, Xin, et al. "3D printing of polymer matrix composites: A review and prospective." Composites Part B: Engineering 110 (2017): 442-458.

17. Whyte, Daniel J., et al. "A review on the challenges of 3D printing of organic powders." Bioprinting 16 (2019): e00057.

18. Banks, Jim. "Adding value in additive manufacturing: researchers in the United Kingdom and Europe look to 3D printing for customization." IEEE pulse 4.6 (2013): 22-26. 
19. Liu, Hao, et al. "An approach to partition workpiece CAD model towards 5-axis support-free 3D printing." The International Journal of Advanced Manufacturing Technology 106.1 (2020): 683699.

20. Guo, Chaofan, Min Zhang, and Bhesh Bhandari. "Model building and slicing in food 3D printing processes: a review." Comprehensive Reviews in Food Science and Food Safety 18.4 (2019): 10521069.

21. Guo, Chaofan, Min Zhang, and Bhesh Bhandari. "Model building and slicing in food 3D printing processes: a review." Comprehensive Reviews in Food Science and Food Safety 18.4 (2019): 10521069.

22. Piedra-Cascón, Wenceslao, et al. "3D printing parameters, supporting structures, slicing, and postprocessing procedures of vat-polymerization additive manufacturing technologies: A narrative review." Journal of Dentistry (2021): 103630.

23. Marro, Alessandro, Taha Bandukwala, and Walter Mak. "Three-dimensional printing and medical imaging: a review of the methods and applications." Current problems in diagnostic radiology 45.1 (2016): 2-9.

24. Bücking, Thore M., et al. "From medical imaging data to 3D printed anatomical models." PloS one 12.5 (2017): e0178540.

25. Kamran, Medhavi, and Abhishek Saxena. "A comprehensive study on 3D printing technology." MIT Int J Mech Eng 6.2 (2016): 63-69.

26. Straub, Jeremy. "Initial work on the characterization of additive manufacturing (3D printing) using software image analysis." Machines 3.2 (2015): 55-71.

27. Jeong, Yoo-Geum, Wan-Sun Lee, and Kyu-Bok Lee. "Accuracy evaluation of dental models manufactured by CAD/CAM milling method and 3D printing method." The journal of advanced prosthodontics 10.3 (2018): 245-251.

28. Guo, Chaofan, Min Zhang, and Bhesh Bhandari. "Model building and slicing in food 3D printing processes: a review." Comprehensive Reviews in Food Science and Food Safety 18.4 (2019): 10521069.

29. Chen, Joshua V., Alan BC Dang, and Alexis Dang. "Comparing cost and print time estimates for six commercially-available 3D printers obtained through slicing software for clinically relevant anatomical models." 3D Printing in Medicine 7.1 (2021): 1-14.

30. Karakurt, Ilbey, and Liwei Lin. "3D printing technologies: Techniques, materials, and postprocessing." Current Opinion in Chemical Engineering 28 (2020): 134-143.

31. Kluska, Ewelina, Piotr Gruda, and NatalIa Majca-NoWaK. "The accuracy and the printing resolution comparison of different 3D printing technologies." Transactions on Aerospace Research 2018.3 (2018): 69-86.

32. Bhavar, Valmik, et al. "A review on powder bed fusion technology of metal additive manufacturing." Additive manufacturing handbook. CRC Press, 2017. 251-253.

33. Shakouri, Taleen, et al. "Comparative study of photoinitiators for the synthesis and 3D printing of a light-curable, degradable polymer for custom-fit hard tissue implants." Biomedical Materials 16.1 (2020): 015007.

34. Ramya, A., and Sai Leela Vanapalli. "3D printing technologies in various applications." International Journal of Mechanical Engineering and Technology 7.3 (2016): 396409. 
35. Constantin, Loic, Zhipeng Wu, Nan Li, Lisha Fan, Jean-François Silvain, and Yong Feng Lu. "Laser 3D printing of complex copper structures." Additive Manufacturing 35 (2020): 101268.

36. Marchment, Taylor, Jay Sanjayan, and Ming Xia. "Method of enhancing interlayer bond strength in construction scale 3D printing with mortar by effective bond area amplification." Materials \& Design 169 (2019): 107684.

37. Usman, Muhammad, et al. "Highly Efficient Permeation and Separation of Gases with MetalOrganic Frameworks Confined in Polymeric Nanochannels." ACS Applied Materials \& Interfaces 12.44 (2020): 49992-50001.

38. Yahya Bozkurt a and Elif Karayel b, "3D printing technology; methods, biomedical," journal of materials research and technology, 18 March 2021

39. L. F. 1, G. Bertrand1, P. Lenormand, D. Grossin and B. Ben-Nissan, "A review of the additive manufacturing (3DP)," HAL archives ouvertes, p. 4, 2019.

40. Morales-Planas, Sergio Morales-Planas and Jordi Lluma-Fuentes, "Multi Jet Fusion PA12 Manufacturing Parameters for Watertightness, Strength and Tolerances," MDPI, 2018

41. Y. L. Tee, "PolyJet 3D Printing of Composite Materials: Experimental and Modelling Approach," Minerals, Metals \& Materials Society, vol. 72(3), no. 13, pp. 3-4, 2020.

42. M. Touri, Fatemeh Kabirian and Mahdi Saadati, "Additive Manufacturing of Biomaterials," The Evolution of Rapid Prototyping, May 10, 2018.

43. W. Harun, N.S. Manam, M.S.I.N. Kamariah and S. Sharif, "A review of powdered additive manufacturing techniques for Ti-6al-4v," elsevier, 7 March 2018

44. T. D. Ngo, Alireza Kashani and, Gabriele Imbalzano, "Additive manufacturing (3D printing): A review of materials, methods, applications and," 6 February 2018.

45. L. Ferrage, Ghislaine Bertrand, Pascal Lenormand and David Grossin, "A review of the additive manufacturing (3DP) of," hal.archives, 31 Jan 2019.

46. M. J. $\Uparrow$ and Abid Haleem, "Additive manufacturing applications in medical cases: A literature based," Alexandria Journal of Medicine, 24 September 2017

47. Y. Bozkurt and Elif Karayel, "3D printing technology; methods, biomedical applications, future opportunities and trends," journal of materials research and technology, 10 July 2021.

48. Rapid prototyping: principles and applications/Chua c.k. Leong K.F. and LIM c/s World scientific publications. Biomedical applications of additive manufacturing: Present and future by Sunpreet Singha and Seeram Ramakrishna Review paper

49. Jones, R.; Haufe, P.; Sells, E.; Iravani, P.; Olliver, V.; Palmer, C.; Bowyer, A. RepRap-The replicating rapid prototyper. Robotica 2011, 29, 177-191. [Google Scholar] [CrossRef]

50. Hinton, T.J.; Jallerat, Q.; Palchesko, R.N.; Park, J.H.; Grodzicki, M.S.; Shue, H.-J.; Ramadan, M.H.; Hudson, A.R.; Feinberg, A.W. Three-dimensional printing of complex biological structures by freeform reversible embedding of suspended hydrogels. Sci. Adv. 2015, 1, e1500758. [Google Scholar] [CrossRef] [Green Version]

51. Feinberg, A.W.; Miller, J.S. Progress in three-dimensional bioprinting. MRS Bull. 2017, 42, 557562. [Google Scholar] [CrossRef] [Green Version]

52. Pusch, K.; Hinton, T.J.; Feinberg, A.W. Large volume syringe pump extruder for desktop 3D printers. HardwareX 2018, 3, 49-61.[Google Scholar] [CrossRef]

53. Livesu, M.; Csiszer, M.; Mehes, E.; Czirok, A. Software tools for cell culture-related 3D printed structures. PLoS ONE 2018, 13, e0203203. [Google Scholar] 
54. Berman B. 3-D printing: The new industrial revolution. Business Horizons. 2012;55(2):15562.[View Article] . [Google Scholar]

55. Liman S. Chest injury due to blunt trauma. European Journal of Cardio-Thoracic Surgery. 2003;23(3):374-8. pmid:12614809 [View Article] [PubMed/NCBI] [Google Scholar] 